\title{
Existence of weak solutions to a certain homogeneous parabolic Neumann problem involving variable exponents and cross-diffusion
}

\author{
Gurusamy Arumugam ${ }^{1}$. André H. Erhardt ${ }^{2}$ (1)
}

Received: 21 April 2020 / Accepted: 30 May 2020 / Published online: 21 June 2020

(ᄋ) The Author(s) 2020

\begin{abstract}
This paper deals with a homogeneous Neumann problem of a nonlinear diffusion system involving variable exponents dependent on spatial and time variables and cross-diffusion terms. We prove the existence of weak solutions using Galerkin's approximation and we derive suitable energy estimates. To this end, we establish the needed Poincaré type inequality for variable exponents related to the Neumann boundary problem. Furthermore, we show that the investigated problem possesses a unique weak solution and satisfies a stability estimate, provided some additional assumptions are fulfilled. In addition, we show under which conditions the solution is nonnegative.
\end{abstract}

Keywords Nonlinear parabolic equations · Existence $\cdot$ Uniqueness $\cdot p(x, t)$-growth $\cdot$ Cross-diffusion

Mathematics Subject Classification 35A01 $35 \mathrm{D} 30 \cdot 35 \mathrm{~K} 65$

\section{Introduction}

In this paper, we study a nonlinear parabolic system with nonstandard growth condition, where the (weak) solution satisfies a homogeneous Neumann boundary condition, which is motivated by several issues and numerous applications. While

Dedicated to Professor Michel Chipot on the occasion of his 70th birthday.

André H. Erhardt

andreerh@math.uio.no

Gurusamy Arumugam

guru.poy@gmail.com

1 Department of Mathematics, National Institute of Technology Calicut, Kattangal, Kerala 673601, India

2 Department of Mathematics, University of Oslo, P.O.Box 1053, Blindern, 0316 Oslo, Norway 
Dirichlet boundary conditions correspond to the perfectly conducting boundary, Neumann boundary conditions correspond to the perfectly isolating boundary (i.e. no-flux boundary condition). We want to highlight that one of the first existence result for a degenerate parabolic Neumann boundary value problem is available in [37]. In the following, we will prove the existence of (weak) solutions to the system we will describe below in detail. Furthermore, we will derive additional assumptions for which this system possesses a unique (weak) solution. Finally, we will establish under which condition the solution is nonnegative.

The investigation of parabolic problems like reaction-diffusion systems or evolutionary equations is motivated amongst others by several applications. For instance, such equations and systems are important for the modelling of space- and timedependent problems, e.g. problems in physics and biology. In particular, evolutionary equations and systems can be used to model physical processes like heat conduction, diffusion processes or wave propagation, see e.g. [10, 27, 48]. The second interesting aspect here is the nonstandard growth setting. Such setting arises for instance by studying certain classes of non-Newtonian fluids such as electro-rheological fluids or fluids with viscosity depending on the temperature. In general, electro-rheological fluids are of high technological interest, because of their ability to change their mechanical properties under the influence of an exterior electromagnetic field [18, 46]. Many electro-rheological fluids are suspensions consisting of solid particles and a carrier oil. These suspensions change their material properties dramatically if they are exposed to an electric field. Known results concern the stationary case with $p(x)$-growth condition, are studied e.g. in $[2,3,17,20,28$, 42]. Furthermore, for the restoration in image processing one also uses some diffusion models with nonstandard growth condition [1, 16, 34, 44]. In the context of parabolic problems with $p(x, t)$-growth, applications are models for flows in porous media [6] or parabolic obstacle problems [21, 23, 24]. Moreover, in the last years parabolic problems with nonstandard growth condition arouse more and more interest in mathematics, cf. [5, 11, 43, 47]. The third interesting aspect of this paper is the effect of a cross-diffusion term. Such a term is for example used to model the interaction between the species, which often leads to cross-diffusion effects $[12,13$, 38]. The difficulty here is that such an effect may lead to unexpected behaviour, see e.g. [15]. Finally, the study of a problem with cross-diffusion is motivated by the fact that parabolic problems with cross-diffusion play a crucial role in biological applications like epidemic diseases, chemotaxis phenomena, cancer growth and population development, cf. [30, 36].

Nowadays, there is a rich literature regarding nonstandard growth problem focused on the existence of (weak) solutions and their properties. For instance, theorems of existence and uniqueness of weak solutions to the prototype problem, i.e. the parabolic $p(x, t)$-Laplacian

$$
u_{t}-\operatorname{div}\left(|\nabla u|^{p(x, t)-2} \nabla u\right)=f,
$$

were proved in [7, 32] for a single equation and in [19] for systems of evolution $p(x, t)$-Laplace equations. The problem with the Cauchy-Dirichlet boundary condition was studied in [25], while in [29] the corresponding Neumann boundary 
problem was considered, see also [40, 49]. Furthermore, in [25] an Aubin-Lions type theorem was established, which we will also use in this paper. In addition, the author of [25] considered more general vector-fields, which are related to the parabolic $p(x, t)$-Laplacian, and inhomogeneities. Moreover, in [26] the existence, uniqueness and stability of a weak solution to the equation

$$
u_{t}-\operatorname{div}(a(x, t, \nabla u))=-\lambda|u|^{p(x, t)-2} u,
$$

where $\lambda \geq 0$ and the vector-field $a(x, t, \cdot)$ satisfies certain $p(x, t)$-growth and monotonicity conditions, cf. [25], was shown, see also [14] for $p=$ constant. Additionally, in [8] it is shown that the solutions of a similar problem may vanish in finite time even if the equation combines the directions of slow and fast diffusion, and the extinction moment is estimated in terms of the data. Further, very recently the existence of weak solutions to a homogeneous Dirichlet problem of a nonlinear diffusion equation involving anisotropic variable exponents and convection was studied in [39].

\subsection{Plan of the paper}

The paper is organised as follows: The rest of this sections is focused on the formulations of the problem, which we are going to study. Furthermore, we will refer some known results on nonstandard Lebesgue and Sobolev spaces, before we state some preliminary results and tools, which are needed to established our existence result. In Sect. 2, we will state our main result. Then, in Sect. 3, we prove the existence of weak solutions to the considered parabolic Neumann boundary problem using Galerkin's approximation and we derive suitable energy estimates. Moreover, in Sect. 4, we will establish under which conditions the weak solution is unique. Finally, in Sect. 5, we will prove that the solution in nonnegative, provided certain assumptions are fulfilled.

\subsection{Notation and formulation of the problem}

In this paper, $\Omega \subset \mathbb{R}^{n}$ denotes a bounded Lipschitz domain of dimension $n \geq 2$ and we write $Q_{T}:=\Omega \times(0, T)$ for the space-time cylinder over $\Omega$ of height $T>0$. Here, $u_{t}$ or $\partial_{t} u$ respectively denote the partial derivative with respect to the time variable $t$ and $\nabla u$ denotes the one with respect to the space variable $x$. Moreover, we denote by $\partial_{\mathcal{P}} Q_{T}:=(\bar{\Omega} \times\{0\}) \cup(\partial \Omega \times(0, T))$ the parabolic boundary of $Q_{T}$ and we write $z=(x, t)$ for points in $\mathbb{R}^{n+1}$. The aim of this paper is the investigation of the following Neumann problem:

$$
\left\{\begin{array}{l}
\partial_{t} u=d_{1} \operatorname{div}\left(A_{1}(x, t, \nabla u)\right)+\operatorname{div}\left(\alpha_{1}(x, t) \nabla u+\alpha_{2}(x, t) \nabla v\right), \quad(x, t) \in Q_{T} \\
\partial_{t} v=d_{2} \operatorname{div}\left(A_{2}(x, t, \nabla v)\right)+\operatorname{div}\left(\alpha_{3}(x, t) \nabla u+\alpha_{4}(x, t) \nabla v\right)-\beta|u|^{q(x, t)-2} u,(x, t) \in Q_{T} \\
\frac{\partial u}{\partial v}=\frac{\partial v}{\partial v}=0,(x, t) \in S_{T} \\
u(x, 0)=u_{0}(x), v(x, 0)=v_{0}(x), x \in \Omega
\end{array}\right.
$$


with $u_{0}, v_{0} \in L^{2}(\Omega)$, where $S_{T}:=\partial \Omega \times(0, T), v$ denotes the exterior normal to the boundary $\partial \Omega$ and $d_{i}>0, i=1,2, \beta \geq 0$ with

$$
u_{\Omega}:=f_{\Omega} u \mathrm{~d} x:=\frac{1}{|\Omega|} \int_{\Omega} u \mathrm{~d} x=0 \quad \text { and } \quad v_{\Omega}=0,
$$

which is the case if

$$
\int_{\Omega} u \mathrm{~d} x=\int_{\Omega} v \mathrm{~d} x=0 .
$$

Furthermore, the vector-fields $A_{i}(x, t, \cdot)$ are assumed to be Carathéodory functions and satisfy the following coercivity, growth and monotonicity conditions:

$$
\begin{gathered}
A_{i}(x, t, \xi) \cdot \xi \geq \mu_{i}|\xi|^{p(x, t)}, \\
\left|A_{i}(x, t, \xi)\right| \leq L_{i}\left(h_{i}+|\xi|^{p(x, t)-1}\right), \\
\left(A_{i}(x, t, \xi)-A_{i}\left(x, t, \xi^{\prime}\right)\right)\left(\xi-\xi^{\prime}\right) \geq 0,
\end{gathered}
$$

where $0<\mu_{i} \leq L_{i}<\infty$, for almost every $(x, t) \in Q_{T}$ and for every $\xi, \xi^{\prime} \in \mathbb{R}^{n}$ with $h_{i} \in L^{p^{\prime}(x, t)}\left(Q_{T}\right)$, where $i=1,2$ and $L^{p^{\prime}(x, t)}\left(Q_{T}\right)$ denotes the nonstandard $p(x, t)$-Lebesgue space for $p^{\prime}(x, t)=\frac{p(x, t)}{p(x, t)-1}$, which we will define later. In addition, the functions $\alpha_{k}(\cdot), k=1, \ldots, 4$ are measurable functions satisfying

$$
0<a_{0} \leq \alpha_{k}(x, t) \leq a_{1}<\infty, a_{0}, a_{1}=\text { constant } \text { for all }(x, t) \in Q_{T} .
$$

Moreover, the growth exponent $p: Q_{T} \rightarrow[2, \infty)$ satisfies the following conditions: There exist constants $\gamma_{1}$ and $\gamma_{2}$, such that

$$
2 \leq \gamma_{1} \leq p(z) \leq \gamma_{2}<\infty \quad \text { and } \quad\left|p\left(z_{1}\right)-p\left(z_{2}\right)\right| \leq \omega\left(d_{\mathcal{P}}\left(z_{1}, z_{2}\right)\right)
$$

hold for any choice of $z_{1}, z_{2} \in Q_{T}$, where $\omega:[0, \infty) \rightarrow[0,1]$ denotes a modulus of continuity. More precisely, we assume that $\omega(\cdot)$ is a concave, non-decreasing function with $\lim _{\rho \downarrow 0} \omega(\rho)=0=\omega(0)$. Moreover, the parabolic distance is given by $d_{\mathcal{P}}\left(z_{1}, z_{2}\right):=\max \left\{\left|x_{1}-x_{2}\right|, \sqrt{\left|t_{1}-t_{2}\right|}\right\}$ for $z_{1}=\left(x_{1}, t_{1}\right), z_{2}=\left(x_{2}, t_{2}\right) \in \mathbb{R}^{n+1}$. In addition, for the modulus of continuity $\omega(\cdot)$ we assume the following weak logarithmic continuity condition

$$
\limsup _{\rho \downarrow 0} \omega(\rho) \log \left(\frac{1}{\rho}\right)<\infty .
$$

Similarly, the exponent $q(x, t)$ is assumed to fulfil the conditions:

$$
1<q(z) \leq 2 \text { and }\left|q\left(z_{1}\right)-q\left(z_{2}\right)\right| \leq \omega\left(d_{\mathcal{P}}\left(z_{1}, z_{2}\right)\right)
$$

for any choice of $z_{1}, z_{2} \in Q_{T}$. 


\subsection{Function spaces}

The spaces $L^{p}(\Omega), W^{1, p}(\Omega)$ and $W_{0}^{1, p}(\Omega)$ denote the usual Lebesgue and Sobolev spaces, while the nonstandard $p(z)$-Lebesgue space $L^{p(z)}\left(Q_{T}, \mathbb{R}^{k}\right)$ is defined as the set of those measurable functions $v: Q_{T} \rightarrow \mathbb{R}^{k}$ for $k \in \mathbb{N}$, which satisfy $|v|^{p(z)} \in L^{1}\left(Q_{T}, \mathbb{R}^{k}\right)$, i.e.

$$
L^{p(z)}\left(Q_{T}, \mathbb{R}^{k}\right):=\left\{v: Q_{T} \rightarrow \mathbb{R}^{k} \text { is measurable in } Q_{T}: \int_{Q_{T}}|v|^{p(z)} \mathrm{d} z<+\infty\right\} .
$$

The set $L^{p(z)}\left(Q_{T}, \mathbb{R}^{k}\right)$ equipped with the Luxemburg norm

$$
\|v\|_{L^{p(z)}\left(Q_{T}\right)}:=\inf \left\{\delta>0: \int_{Q_{T}}\left|\frac{v}{\delta}\right|^{p(z)} \mathrm{d} z \leq 1\right\}
$$

becomes a Banach space. This space is separable and reflexive, see [4, 19]. For elements of $L^{p(z)}\left(Q_{T}, \mathbb{R}^{k}\right)$ the generalised Hölder's inequality holds in the following form: If $f \in L^{p(z)}\left(Q_{T}, \mathbb{R}^{k}\right)$ and $g \in L^{p^{\prime}(z)}\left(Q_{T}, \mathbb{R}^{k}\right)$, where $p^{\prime}(z)=\frac{p(z)}{p(z)-1}$, we have

$$
\left|\int_{Q_{T}} f g \mathrm{~d} z\right| \leq\left(\frac{1}{\gamma_{1}}+\frac{\gamma_{2}-1}{\gamma_{2}}\right)\|f\|_{L^{p(z)}\left(Q_{T}\right)}\|g\|_{L^{p^{\prime}(z)}\left(Q_{T}\right)},
$$

see also [4]. Moreover, the norm $\|\cdot\|_{L^{p(z)}\left(Q_{T}\right)}$ can be estimated as follows

$$
-1+\|v\|_{L^{p(z)}\left(Q_{T}\right)}^{\gamma_{1}} \leq \int_{Q_{T}}|v|^{p(z)} \mathrm{d} z \leq\|v\|_{L^{p(z)}\left(Q_{T}\right)}^{\gamma_{2}}+1 .
$$

Notice that we will use also the abbreviation $p(\cdot)$ for the exponent $p(z)$. Next, we introduce nonstandard Sobolev spaces for fixed $t \in(0, T)$. From assumption (1.8) we know that $p(\cdot, t)$ satisfy $\left|p\left(x_{1}, t\right)-p\left(x_{2}, t\right)\right| \leq \omega\left(\left|x_{1}-x_{2}\right|\right)$ for any choice of $x_{1}, x_{2} \in \Omega$ and for every $t \in(0, T)$. Then, we define for every fixed $t \in(0, T)$ the Banach space $W^{1, p(\cdot, t)}(\Omega)$ as

$$
W^{1, p(\cdot t)}(\Omega):=\left\{u \in L^{p(\cdot, t)}(\Omega, \mathbb{R}) \mid \nabla u \in L^{p(\cdot, t)}\left(\Omega, \mathbb{R}^{n}\right)\right\}
$$

equipped with the norm

$$
\|u\|_{W^{1, p(\cdot, t)}(\Omega)}:=\|u\|_{L^{p(, t)}(\Omega)}+\|\nabla u\|_{L^{p(t, t)}(\Omega)} .
$$

In addition, we define $W_{0}^{1, p(\cdot, t)}(\Omega)$ as the closure of $C_{0}^{\infty}(\Omega)$ in $W^{1, p(\cdot, t)}(\Omega)$ and we denote by $W^{1, p(\cdot, t)}(\Omega)^{\prime}$ its dual. For every $t \in(0, T)$ the inclusion $W_{0}^{1, p(\cdot t)}(\Omega) \subset W_{0}^{1, \gamma_{1}}(\Omega)$ holds true. Furthermore, we denote by $W_{g}^{p(\cdot)}\left(Q_{T}\right)$ the Banach space

$$
W_{g}^{p(\cdot)}\left(Q_{T}\right):=\left\{u \in\left[g+L^{1}\left(0, T ; W_{0}^{1,1}(\Omega)\right)\right] \cap L^{p(\cdot)}\left(Q_{T}\right) \mid \nabla u \in L^{p(\cdot)}\left(Q_{T}, \mathbb{R}^{n}\right)\right\}
$$

equipped with the norm $\|u\|_{W^{p(\cdot)}\left(Q_{T}\right)}:=\|u\|_{L^{p(\cdot)}\left(Q_{T}\right)}+\|\nabla u\|_{L^{p(\cdot)}\left(Q_{T}\right)}$. If $g=0$ we write $W_{0}^{p(\cdot)}\left(Q_{T}\right)$ instead of $W_{g}^{p(\cdot)}\left(Q_{T}\right)$. Here, it is worth to mention that the notion $(u-g) \stackrel{\in}{\in} W_{0}^{p(\cdot)}\left(Q_{T}\right)$ or $u \in g+W_{0}^{p(\cdot)}\left(Q_{T}\right)$ respectively indicate that $u$ agrees with $g$ 
on the lateral boundary of the cylinder $Q_{T}$, i.e. $u \in W_{g}^{p(\cdot)}\left(Q_{T}\right)$. In addition, we denote by $W^{p(\cdot)}\left(Q_{T}\right)^{\prime}$ the dual of the space $W_{0}^{p(\cdot)}\left(Q_{T}\right)$. Note that if $v \in W^{p(\cdot)}\left(Q_{T}\right)^{\prime}$, then there exist functions $v_{i} \in L^{p^{\prime}(\cdot)}\left(Q_{T}\right), i=0,1, \ldots, n$, such that

$$
\langle\langle v, w\rangle\rangle_{Q_{T}}=\int_{Q_{T}}\left(v_{0} w+\sum_{i=1}^{n} v_{i} \nabla_{i} w\right) \mathrm{d} z
$$

for all $w \in W_{0}^{p(\cdot)}\left(Q_{T}\right)$. Furthermore, if $v \in W^{p(\cdot)}\left(Q_{T}\right)^{\prime}$, we define the norm

$$
\|v\|_{W^{p(\cdot)}\left(Q_{T}\right)^{\prime}}:=\sup \left\{\langle\langle v, w\rangle\rangle_{Q_{T}} \mid w \in W_{0}^{p(\cdot)}\left(Q_{T}\right),\|w\|_{W_{0}^{p(\cdot)}\left(Q_{T}\right)} \leq 1\right\} .
$$

Notice, whenever (1.13) holds, we can write $v=v_{0}-\sum_{i=1}^{n} \nabla_{i} v_{i}$, where $\nabla_{i} v_{i}$ has to be interpreted as a distributional derivate. By

$$
w \in W\left(Q_{T}\right):=\left\{w \in W^{p(\cdot)}\left(Q_{T}\right) \mid w_{t} \in W^{p(\cdot)}\left(Q_{T}\right)^{\prime}\right\}
$$

we mean that there exists $w_{t} \in W^{p(\cdot)}\left(Q_{T}\right)^{\prime}$, such that

$$
\left\langle\left\langle w_{t}, \varphi\right\rangle\right\rangle_{Q_{T}}=-\int_{Q_{T}} w \cdot \varphi_{t} \mathrm{~d} z \text { for all } \varphi \in C_{0}^{\infty}\left(Q_{T}\right),
$$

see also [19]. The previous equality makes sense due to the inclusions

$$
W^{p(\cdot)}\left(Q_{T}\right) \hookrightarrow L^{2}\left(Q_{T}\right) \cong\left(L^{2}\left(Q_{T}\right)\right)^{\prime} \hookrightarrow W^{p(\cdot)}\left(Q_{T}\right)^{\prime}
$$

which allow us to identify $w$ as an element of $W^{p(\cdot)}\left(Q_{T}\right)^{\prime}$. Finally, we are in the situation to give the definition of a weak solution to the parabolic problem (1.1):

Definition 1.1 A pair of function $(u, v)$ is called a weak solution of (1.1) if and only if $(u, v) \in\left(L^{\infty}\left(0, T ; L^{2}(\Omega)\right) \cap W^{p(\cdot)}\left(Q_{T}\right)\right)^{2}$ and for every test function $\phi_{i} \in C_{0}^{\infty}(\bar{\Omega} \times[0, T)), i=1,2$, the following equalities hold:

$$
\begin{aligned}
& \int_{Q_{T}} u \frac{\partial \phi_{1}}{\partial t}-\left[d_{1} A_{1}(x, t, \nabla u)+\alpha_{1}(x, t) \nabla u+\alpha_{2}(x, t) \nabla v\right] \cdot \nabla \phi_{1} \mathrm{~d} z=\left.\int_{\Omega} u \phi_{1} \mathrm{~d} x\right|_{0} ^{T} \\
& \int_{Q_{T}} v \frac{\partial \phi_{2}}{\partial t}-\left[d_{2} A_{2}(x, t, \nabla v)+\alpha_{3}(x, t) \nabla u+\alpha_{4}(x, t) \nabla v\right] \cdot \nabla \phi_{2}-\beta|u|^{q(x, t)-2} u \phi_{2} \mathrm{~d} z \\
& =\left.\int_{\Omega} v \phi_{2} \mathrm{~d} x\right|_{0} ^{T}
\end{aligned}
$$

where (1.3) and the initial value conditions $u(\cdot, 0)=u_{0}(x) \in L^{2}(\Omega)$, $v(\cdot, 0)=v_{0}(x) \in L^{2}(\Omega)$ a.e. on $\Omega$, i.e.

$$
\frac{1}{h} \int_{0}^{h} \int_{\Omega}\left|u-u_{0}\right|^{2} \mathrm{~d} x \mathrm{~d} t \rightarrow 0 \text { and } \frac{1}{h} \int_{0}^{h} \int_{\Omega}\left|v-v_{0}\right|^{2} \mathrm{~d} x \mathrm{~d} t \rightarrow 0 \quad \text { as } h \downarrow 0
$$


are fulfilled.

\subsection{Preliminary results and tools}

To derive our existence result, we will need the following Poincaré type estimate, which is a modification of the Poincaré type estimate from [22, Lemma 3.9].

Lemma 1.2 Let $\Omega \subset \mathbb{R}^{n}, n \geq 2$, be a bounded Lipschitz domain. Assume that $u \in L^{\infty}\left(0, T ; L^{2}(\Omega)\right) \cap W^{p(\cdot)}\left(Q_{T}\right)$ with $u_{\Omega}=0$ and $p(\cdot)$ satisfies the conditions (1.8) and (1.9). Then, there exists a constant $c=c\left(n, \gamma_{1}, \gamma_{2}, \operatorname{diam}(\Omega), \omega(\cdot)\right)$, such that the following two Poincaré type estimates are valid:

$$
\int_{Q_{T}}|u|^{p(\cdot)} \mathrm{d} z \leq c\left(\|u\|_{L^{\infty}\left(0, T ; L^{2}(\Omega)\right)}^{\frac{4 \gamma_{2}}{n+2}}+1\right)\left(\int_{Q_{T}}|\nabla u|^{p(\cdot)}+1 \mathrm{~d} z\right)
$$

and

$$
\|u\|_{L^{p(z)}\left(Q_{T}\right)}^{\gamma_{1}} \leq c\left(\|u\|_{L^{\infty}\left(0, T ; L^{2}(\Omega)\right)}^{\frac{4 \gamma_{2}}{n+2}}+1\right)\left(\int_{Q_{T}}|\nabla u|^{p(\cdot)}+1 \mathrm{~d} z\right) .
$$

Proof The proof of Lemma 1.2 is very similar to the proof of [22, Lemma 3.9]. To derive the needed Poincaré type estimate we apply the Gagliardo-Nirenberg's inequality from [41]. Then, we have to follow the proof of [22, Lemma 3.9] to derive the following estimate:

$$
\int_{Q_{T}}|u|^{p(\cdot)} \mathrm{d} z \leq c\left(\|u\|_{L^{\infty}\left(0, T ; L^{2}(\Omega)\right)}^{\frac{4 \gamma_{2}}{n+2}}+1\right)\left(\int_{Q_{T}}|\nabla u|^{p(\cdot)}+|u|^{\gamma_{1}}+1 \mathrm{~d} z\right)
$$

with a constant $c=c\left(n, \gamma_{1}, \gamma_{2}, \omega(\cdot)\right)$. Notice that up to (1.18) both proofs are identically, the only difference is that we now have to apply

$$
\left\|u-u_{\Omega}\right\|_{L^{\gamma_{1}(\Omega)}} \leq c_{p}\|\nabla u\|_{L^{\gamma_{1}(\Omega)}}
$$

due to the Neumann boundary condition, where $c_{p}=c_{p}\left(n, \gamma_{1}, \operatorname{diam}(\cdot)\right)$ and we have to use (1.2). Thus, we can estimate as follows

$$
\int_{Q_{T}}|u|^{\gamma_{1}} \mathrm{~d} z \leq c_{p}\left(n, \gamma_{1}, \operatorname{diam}(\cdot)\right) \int_{Q_{T}}|\nabla u|^{p(\cdot)}+1 \mathrm{~d} z,
$$

which proves (1.16). To complete the proof we now have to combine (1.16) and (1.12), which implies (1.17).

Remark 1.3 Notice that Lemma 1.2 is valid for a exponent function $p: Q_{T} \rightarrow\left(\frac{2 n}{n+2}, \infty\right)$, while the problem (1.1) requires the restriction $p(x, t) \geq 2$ due to the cross-diffusion terms. 
After proving the energy estimate for the (weak) solutions, we will derive from Lemma 1.2 the needed $L^{p(\cdot)}\left(Q_{T}\right)$-bounds for the approximate solution to (1.1). This together with the following Aubin-Lions type Theorem [25, Theorem 1.3] will guarantee the strong convergence of the approximate solution to the solution in $L^{p(\cdot)}\left(Q_{T}\right)$. The Aubin-Lions type Theorem reads as follows:

Theorem 1.4 Let $\Omega \subset \mathbb{R}^{n}$ be an open, bounded Lipschitz domain with $n \geq 2$ and $p(\cdot)>\frac{2 n}{n+2}$ satisfying (1.8) and (1.9). Furthermore, define $\hat{p}(\cdot):=\max \{2, p(\cdot)\}$. Then, the inclusion $W\left(Q_{T}\right) \hookrightarrow L^{\hat{p}(\cdot)}\left(Q_{T}\right)$ is compact.

Moreover, the next two lemmas, which are useful tools when dealing with $p$-growth problems, we will need to prove the uniqueness of the weak solution to system (1.1). Therefore, we define a function by

$$
V_{\mu, \mathfrak{p}}(A):=\left(\mu^{2}+|A|^{2}\right)^{\frac{\mathfrak{p}}{2}} A \text { for } A \in \mathbb{R}^{k}, \mathfrak{p}>-1 \text { and } \mu \geq 0 .
$$

Moreover, we cite the following lemma from [33, Lemma 2.1], which is established for the case $\mathfrak{p} \geq 0$ in [31] and in the case $0>\mathfrak{p}>-1$ in [33].

Lemma 1.5 There exists a positive constant, depending on $\mathfrak{p}>-1$, such that for all $A, B \in \mathbb{R}^{k}$ with $A \neq B$, we have

$$
\frac{1}{c}\left(\mu^{2}+|A|^{2}+|B|^{2}\right)^{\frac{\mathfrak{p}}{2}}|A-B| \leq\left|V_{\mu, \mathfrak{p}}(A)-V_{\mu, \mathfrak{p}}(B)\right| \leq c\left(\mu^{2}+|A|^{2}+|B|^{2}\right)^{\frac{\mathfrak{p}}{2}}|A-B|
$$

with $\mu \geq 0$.

Since $q(\cdot)>1$, we are able to choose $\mathfrak{p}=q(\cdot)-2>-1$. Choosing $\mu=0$ and $k=1$, then we consider $V(A)=|A|^{q(\cdot)-2} A$. This allows to infer from Lemma 1.5 the next lemma.

Lemma 1.6 There exists a constant $c:=c\left(n, \gamma_{1}, \gamma_{2}\right)$, such that for any $A, B \in \mathbb{R}^{k}$ and $1<q(\cdot) \leq 2$, there holds

$$
\frac{1}{c}\left(|A|^{2}+|B|^{2}\right)^{\frac{q(\cdot)-2}{2}}|A-B| \leq|V(A)-V(B)| \leq c\left(|A|^{2}+|B|^{2}\right)^{\frac{q(\cdot)-2}{2}}|A-B|
$$

and

$$
\left(|A|^{2}+|B|^{2}\right)^{\frac{q(\cdot)-2}{2}}|A-B|^{2} \leq c(V(A)-V(B)) \cdot(A-B),
$$

where $A \neq B$.

We are using Lemma 1.6 only for $1<q(\cdot) \leq 2$. However, Lemma 1.6 holds true for all $1<q(\cdot)$. 


\section{Statement of results}

In this section we state the main results of this paper. The existence result reads as follows:

Theorem 2.1 Let $\Omega \subset \mathbb{R}^{n}$ be an open, bounded Lipschitz domain with $n \geq 2$, $d_{i}>0, i=1,2, \beta \geq 0$ and $u(x, 0)=u_{0}(x) \in L^{2}(\Omega), v(x, 0)=v_{0}(x) \in L^{2}(\Omega), x \in \Omega$, where the initial values are given. Furthermore, suppose that growth exponent $p: Q_{T} \rightarrow[2, \infty)$ satisfies $(1.8)$ and $(1.9)$, while $q: Q_{T} \rightarrow(1,2]$ satisfies $(1.10)$ and (1.9). In addition, assume that the vector-fields $A_{i}(x, t, \cdot)$ are Carathéodory functions and satisfy the coercivity (1.4), growth (1.5) and monotonicity (1.6) conditions. Moreover, let $\alpha_{k}(\cdot), k=1, \ldots, 4$ be measurable functions satisfying (1.7). Then, there exists at least one (weak) solution $(u, v) \in\left(L^{\infty}\left(0, T ; L^{2}(\Omega)\right) \cap W^{p(\cdot)}\left(Q_{T}\right)\right)^{2}$ with $\left(u_{t}, v_{t}\right) \in\left(W^{p(\cdot)}\left(Q_{T}\right)^{\prime}\right)^{2}$ and $u_{\Omega}=v_{\Omega}=0, c f$. (1.2) or (1.3), to the homogeneous Neumann problem (1.1), which satisfies the following energy estimate:

$$
\sup _{0 \leq t \leq T} \int_{\Omega}|u(\cdot, t)|^{2}+|v(\cdot, t)|^{2} \mathrm{~d} x+\int_{Q_{T}}|\nabla u|^{2}+|\nabla v|^{2}+|\nabla u|^{p(x, t)}+|\nabla v|^{p(x, t)} \mathrm{d} z \leq c
$$

with a constant $c=c\left(a_{0}, a_{1}, d_{1}, d_{2}, \beta, \mu_{1}, \mu_{2}, \gamma_{1}, \gamma_{2},\left\|u_{0}\right\|_{L^{2}(\Omega)},\left\|v_{0}\right\|_{L^{2}(\Omega)},\left|Q_{T}\right|\right)$.

Furthermore, the solution to the homogeneous Neumann problem (1.1) possesses a unique (weak) solution under certain assumption. The result reads as follows:

Theorem 2.2 Suppose that either $q(\cdot) \equiv 2$ or $\beta \equiv 0$. Under the assumptions of Theorem 2.1

i) and the additional assumption

$$
\left(A_{i}(x, t, \xi)-A_{i}\left(x, t, \xi^{\prime}\right)\right)\left(\xi-\xi^{\prime}\right) \geq \mu_{i}\left|\xi-\xi^{\prime}\right|^{2}, \quad i=1,2,
$$

for almost every $(x, t) \in Q_{T}$ and for every $\xi, \xi^{\prime} \in \mathbb{R}^{n}$ the (weak) solution to the homogeneous Neumann problem (1.1) is unique, provided that

$$
a_{0}-a_{1}+\min \left\{d_{1} \mu_{1}, d_{2} \mu_{2}\right\} \geq 0 .
$$

ii) and in case that for $k=1, \ldots, 4$ we have

$$
\alpha_{k}(x, t)=a_{0}=\text { constant }
$$

system (1.1) possesses a unique weak solution without further additional assumptions.

iii) and in case that we have

$$
0<a_{k_{0}} \leq \alpha_{k}(x, t) \leq a_{k_{1}}<\infty \text {, with } a_{k_{0}}, a_{k_{1}}=\text { constant }
$$

for all $k=1, \ldots, 4$ and $(x, t) \in Q_{T}$, and additionally 


$$
a_{2_{1}}+a_{3_{1}} \leq \min \left\{a_{1_{0}}, a_{4_{0}}\right\}
$$

is satisfied, then system (1.1) possesses a unique weak solution.

Please compare the uniqueness result from [10], here a similar restriction occurs due to the term $\beta|u|^{q(\cdot)-2} u$. In addition, one can conclude from the proof of the Theorem 2.2 immediately the following stability result:

Lemma 2.3 Under the assumptions of Theorem 2.2 with $\beta=0$, two unique weak solutions $(u, v)$ and $\left(u_{1}, v_{1}\right)$ to system (1.1) with the different initial values $\left(u_{0}, v_{0}\right) \in\left(L^{2}(\Omega)\right)^{2}$ and $\left(u_{1_{0}}, v_{1_{0}}\right) \in\left(L^{2}(\Omega)\right)^{2}$ satisfy the following stability estimate:

$$
\left\|u(x, t)-u_{1}(x, t)\right\|_{L^{2}(\Omega)}^{2}+\left\|v(x, t)-v_{1}(x, t)\right\|_{L^{2}(\Omega)}^{2} \leq\left\|u_{0}(x)-u_{1_{0}}(x)\right\|_{L^{2}(\Omega)}^{2}+\left\|v_{0}(x)-v_{1_{0}}(x)\right\|_{L^{2}(\Omega)}^{2}
$$

for all every $t \in[0, T)$, i.e. the solutions are controlled by their initial values completely.

Finally, we will show under which conditions the (weak) solution to the homogeneous Neumann problem (1.1) is nonnegative. The result reads as follows

Theorem 2.4 Under the assumptions of Theorem 2.1 and the additional assumption that the initial values $u_{0}(x) \in L^{2}(\Omega)$ and $v_{0}(x) \in L^{2}(\Omega)$ are nonnegative, i.e. $u_{0}(x) \geq 0$ and $v_{0}(x) \geq 0$, then the solution itself is nonnegative, provided either condition (2.4) or condition (2.5) with (2.6) is satisfied. Furthermore, in both cases this solution is unique due to Theorem 2.2 , provided $q(\cdot) \equiv 2$ or $\beta=0$.

\section{Proof of the existence result}

In this section, we prove our existence result utilising Galerkin's approximations, cf. $[9,25,50]$.

Proof of Theorem 2.1 The construction of a sequence of Galerkin's approximations is as follows: First of all, we want to recall that $\Omega \subseteq \mathbb{R}^{n}$ is an open, bounded Lipschitz domain and due to the dense embeddings $W^{1, s}(\Omega) \subset L^{2}(\Omega)$ and $\left(L^{2}(\Omega)\right)^{\prime} \subset W^{-1, s^{\prime}}(\Omega)$ one has the inclusions

$$
W^{1, s}(\Omega) \hookrightarrow L^{2}(\Omega) \cong\left(L^{2}(\Omega)\right)^{\prime} \hookrightarrow W^{-1, s^{\prime}}(\Omega),
$$

where the injections are compact. Note that $W_{0}^{1, s}(\Omega) \subset W^{1, s}(\Omega)$ also holds true. Furthermore, it is known that for $1<\gamma_{1} \leq s \leq \gamma_{2}<\infty$ the space $L^{s}(\Omega)$ is a separable and reflexive Banach space, and similarly, $W^{1, s}(\Omega)$ is a separable and reflexive Banach space. In the case of Dirichlet boundary values one would consider $\left\{w_{i}(x)\right\}_{i=1}^{\infty} \subset W_{0}^{1, \gamma_{2}}(\Omega) \subset W^{1, \gamma_{2}}(\Omega)$, which is an orthonormal basis in $L^{2}(\Omega)$, while 
here one can follow the approach from [13], i.e. one considers the spectral problem: Find $f \in W^{1,2}(\Omega)$ and $\lambda \in \mathbb{R}$ such that

$$
\begin{cases}\langle\nabla f, \nabla \eta\rangle=\lambda\langle f, \eta\rangle & \text { for all } \eta \in W^{1,2}(\Omega) \\ \nabla f \cdot \hat{v}=0 & \text { on } \partial \Omega\end{cases}
$$

where $\hat{v}$ is the unit outward normal. Then, problem (3.1) possesses a sequence of nondecreasing eigenvalues $\left\{\lambda_{i}\right\}_{i=1}^{\infty}$ and a sequence of corresponding eigenfunctions $\left\{w_{i}(x)\right\}_{i=1}^{\infty}$ forming an orthogonal basis in $W^{1,2}(\Omega)$ and an orthonormal basis in $L^{2}(\Omega)$ $\left(W^{1, \gamma_{2}}(\Omega) \subset W^{1, \gamma_{1}}(\Omega) \subseteq W^{1,2}(\Omega) \subset L^{2}(\Omega)\right)$, see also [35]. Next, fix a positive integer $m$ and define the approximate solution to problem (1.1) in the following way:

$$
u^{(m)}(z):=\sum_{i=1}^{m} c_{i}^{(m)}(t) w_{i}(x) \quad \text { and } \quad v^{(m)}(z):=\sum_{i=1}^{m} d_{i}^{(m)}(t) w_{i}(x)
$$

where the coefficients $c_{i}^{(m)}(t)$ and $d_{i}^{(m)}(t)$ are defined via the identities

$$
\int_{\Omega} u_{t}^{(m)} w_{i}(x)+\left[d_{1} A_{1}\left(x, t, \nabla u^{(m)}\right)+\alpha_{1}(x, t) \nabla u^{(m)}+\alpha_{2}(x, t) \nabla v^{(m)}\right] \nabla w_{i}(x) \mathrm{d} x=0
$$

and

$$
\begin{aligned}
\int_{\Omega} v_{t}^{(m)} w_{i}(x) & +\left[d_{2} A_{2}\left(x, t, \nabla v^{(m)}\right)+\alpha_{3}(x, t) \nabla u^{(m)}+\alpha_{4}(x, t) \nabla v^{(m)}\right] \nabla w_{i}(x) \mathrm{d} x \\
& =-\beta \int_{\Omega}\left|u^{(m)}\right|^{q(\cdot)-2} u^{(m)} \cdot w_{i}(x) \mathrm{d} x
\end{aligned}
$$

for $i=1, \ldots, m$ and $t \in(0, T)$ with the initial conditions

$$
c_{i}^{(m)}(0)=\int_{\Omega} u_{0}(x) w_{i}(x) \mathrm{d} x \quad \text { and } \quad d_{i}^{(m)}(0)=\int_{\Omega} v_{0}(x) w_{i}(x) \mathrm{d} x .
$$

Then, this generates a system of $2 m$ ordinary differential equations

$$
\begin{aligned}
& \left(c_{i}^{(m)}(t)\right)^{\prime}=F_{i}\left(t, c_{1}^{(m)}(t), \ldots, c_{m}^{(m)}(t), d_{1}^{(m)}(t), \ldots, d_{m}^{(m)}(t)\right), \quad c_{i}^{(m)}(0)=\int_{\Omega} u_{0}(x) w_{i}(x) \mathrm{d} x, \\
& \left(d_{i}^{(m)}(t)\right)^{\prime}=G_{i}\left(t, c_{1}^{(m)}(t), \ldots, c_{m}^{(m)}(t), d_{1}^{(m)}(t), \ldots, d_{m}^{(m)}(t)\right), \quad d_{i}^{(m)}(0)=\int_{\Omega} v_{0}(x) w_{i}(x) \mathrm{d} x,
\end{aligned}
$$

By [45, Theorem 1.44, p. 25] we know that, there is for every finite system (3.4) a solution $\left(c_{i}^{(m)}(t), d_{i}^{(m)}(t)\right), i=1, \ldots, m$ on the interval $\left(0, T_{m}\right) \subset(0, T)$ for some $T_{m}>0$. Therefore, we multiply equation (3.2) by the coefficients $c_{i}^{(m)}(t)$ and equation (3.3) by $d_{i}^{(m)}(t)$. Then, integrating the resulting equations over $(0, \tau)$ for an arbitrarily $\tau \in\left(0, T_{m}\right)$ and summing them over $i=1, \ldots, m$. This yields 


$$
\begin{aligned}
& \int_{Q_{\tau}} u_{t}^{(m)} u^{(m)}+\left[d_{1} A_{1}\left(x, t, \nabla u^{(m)}\right)+\alpha_{1}(x, t) \nabla u^{(m)}+\alpha_{2}(x, t) \nabla v^{(m)}\right] \nabla u^{(m)} \mathrm{d} z=0, \\
& \int_{Q_{\tau}} v_{t}^{(m)} v^{(m)}+\left[d_{2} A_{2}\left(x, t, \nabla v^{(m)}\right)+\alpha_{3}(x, t) \nabla u^{(m)}+\alpha_{4}(x, t) \nabla v^{(m)}\right] \nabla v^{(m)} \mathrm{d} z \\
& =-\beta \int_{Q_{\tau}}\left|u^{(m)}\right|^{q(\cdot)-2} u^{(m)} \cdot v^{(m)} \mathrm{d} z,
\end{aligned}
$$

for a.e. $\tau \in\left(0, T_{m}\right)$. Furthermore, we can conclude the following estimate by applying the conditions (1.4) and (1.7):

$$
\begin{aligned}
& \frac{1}{2} \int_{0}^{\tau}\left(\partial_{t} \int_{\Omega}\left|u^{(m)}\right|^{2}+\left|v^{(m)}\right|^{2} \mathrm{~d} x\right) \mathrm{d} t+a_{0} \int_{Q_{\tau}}\left|\nabla u^{(m)}\right|^{2}+\left|\nabla v^{(m)}\right|^{2} \mathrm{~d} z \\
& \quad+\min \left\{d_{1} \mu_{1}, d_{2} \mu_{2}\right\} \int_{Q_{\tau}}\left|\nabla u^{(m)}\right|^{p(x, t)}+\left|\nabla v^{(m)}\right|^{p(x, t)} \mathrm{d} z \\
& \leq-\int_{Q_{\tau}}\left(\alpha_{2}(x, t)+\alpha_{3}(x, t)\right) \nabla u^{(m)} \nabla v^{(m)} \mathrm{d} z-\beta \int_{Q_{\tau}}\left|u^{(m)}\right|^{q(\cdot)-2} u^{(m)} \cdot v^{(m)} \mathrm{d} z \\
& \leq 2 a_{1} \int_{Q_{\tau}}\left|\nabla u^{(m)}\right|\left|\nabla v^{(m)}\right| \mathrm{d} z+\beta \int_{Q_{\tau}}\left|u^{(m)}\right|^{q(\cdot)-1}\left|v^{(m)}\right| \mathrm{d} z
\end{aligned}
$$

for a.e. $\tau \in\left(0, T_{m}\right)$. Please notice that in the case $p(\cdot) \equiv 2$ we can immediately absorb the term

$$
2 a_{1} \int_{Q_{\tau}}\left|\nabla u^{(m)}\right|\left|\nabla v^{(m)}\right| \mathrm{d} z
$$

on the left-hand side of the previous estimate using Cauchy's inequality, provided

$$
\bar{c}_{*}:=a_{0}-a_{1}+\min \left\{d_{1} \mu_{1}, d_{2} \mu_{2}\right\} \geq 0,
$$

which finally yields

$$
\frac{1}{2} \int_{0}^{\tau}\left(\partial_{t} \int_{\Omega}\left|u^{(m)}\right|^{2}+\left|v^{(m)}\right|^{2} \mathrm{~d} x\right) \mathrm{d} t+\bar{c}_{*} \int_{Q_{\tau}}\left|\nabla u^{(m)}\right|^{2}+\left|\nabla v^{(m)}\right|^{2} \mathrm{~d} z \leq \beta \int_{Q_{\tau}}\left|u^{(m)}\right|^{q(\cdot)-1}\left|v^{(m)}\right| \mathrm{d} z
$$

for a.e. $\tau \in\left(0, T_{m}\right)$. In case that $p(\cdot) \geq \gamma_{1}>2$, we have to utilise Hölder's inequality and Cauchy's inequality to get the following inequality

$$
\begin{aligned}
2 a_{1} \int_{Q_{\tau}}\left|\nabla u^{(m)}\right|\left|\nabla v^{(m)}\right| \mathrm{d} z & \leq 2 a_{1}\left(\int_{Q_{\tau}}\left|\nabla u^{(m)}\right|^{2} \mathrm{~d} z\right)^{\frac{1}{2}}\left(\int_{Q_{\tau}}\left|\nabla v^{(m)}\right|^{2} \mathrm{~d} z\right)^{\frac{1}{2}} \\
& =\left(\frac{4 a_{1}^{2}}{a_{0}} \int_{Q_{\tau}}\left|\nabla u^{(m)}\right|^{2} \mathrm{~d} z\right)^{\frac{1}{2}}\left(a_{0} \int_{Q_{\tau}}\left|\nabla v^{(m)}\right|^{2} \mathrm{~d} z\right)^{\frac{1}{2}} \\
& \leq \frac{2 a_{1}^{2}}{a_{0}} \int_{Q_{\tau}}\left|\nabla u^{(m)}\right|^{2} \mathrm{~d} z+\frac{a_{0}}{2} \int_{Q_{\tau}}\left|\nabla v^{(m)}\right|^{2} \mathrm{~d} z .
\end{aligned}
$$


Furthermore, by Young's inequality with $2 / p(\cdot)+(p(\cdot)-2) / p(\cdot)=1$, we can estimate as follows

$$
\begin{aligned}
\frac{2 a_{1}^{2}}{a_{0}} \int_{Q_{\tau}}\left|\nabla u^{(m)}\right|^{2} \mathrm{~d} z & =\int_{Q_{\tau}} \frac{2 a_{1}^{2}}{a_{0}}\left(\frac{2}{\min \left\{d_{1} \mu_{1}, d_{2} \mu_{2}\right\}}\right)^{\frac{2}{p())}}\left(\left(\frac{\min \left\{d_{1} \mu_{1}, d_{2} \mu_{2}\right\}}{2}\right)^{\frac{1}{p(-)}}\left|\nabla u^{(m)}\right|\right)^{2} \mathrm{~d} z \\
& \leq \frac{\min \left\{d_{1} \mu_{1}, d_{2} \mu_{2}\right\}}{\gamma_{1}} \int_{Q_{\tau}}\left|\nabla u^{(m)}\right|^{p(x, t)} \mathrm{d} z+\bar{c}_{\dagger}\left|Q_{\tau}\right|,
\end{aligned}
$$

where

$$
\bar{c}_{\dagger}:=\frac{\gamma_{2}}{\gamma_{1}-2} \max \left\{\left(\frac{2 a_{1}^{2}}{a_{0}}\right)^{\frac{\gamma_{2}}{\gamma_{1}-2}},\left(\frac{2 a_{1}^{2}}{a_{0}}\right)^{\frac{\gamma_{1}}{\gamma_{2}-2}}\right\} \max \left\{\theta^{\frac{2}{\gamma_{1}-2}}, \theta^{\frac{2}{\gamma_{2}-2}}\right\}
$$

with $\theta:=2 / \min \left\{d_{1} \mu_{1}, d_{2} \mu_{2}\right\}$. This implies

$$
\begin{aligned}
\int_{0}^{\tau}\left(\partial_{t} \int_{\Omega}\left|u^{(m)}\right|^{2}+\left|v^{(m)}\right|^{2} \mathrm{~d} x\right) \mathrm{d} t & +\int_{Q_{\tau}}\left|\nabla u^{(m)}\right|^{2}+\left|\nabla v^{(m)}\right|^{2}+\left|\nabla u^{(m)}\right|^{p(\cdot)}+\left|\nabla v^{(m)}\right|^{p(\cdot)} \mathrm{d} z \\
& \leq \bar{c}_{\ddagger}\left(\beta \int_{Q_{\tau}}\left|u^{(m)}\right|^{q(\cdot)-1}\left|v^{(m)}\right| \mathrm{d} z+\bar{c}_{\dagger}\left|Q_{\tau}\right|\right)
\end{aligned}
$$

for a.e. $\tau \in\left(0, T_{m}\right)$, where

$$
\bar{c}_{\ddagger}:=\frac{2 \gamma_{1}}{\gamma_{1}-1} \max \left\{1, \frac{1}{\min \left\{a_{0}, d_{1} \mu_{1}, d_{2} \mu_{2}\right\}}\right\} .
$$

Furthermore, for all $p(\cdot)$ satisfying (1.8) and (1.9) we have by Cauchy's inequality the following:

$$
\begin{aligned}
\int_{0}^{\tau}\left(\partial_{t} \int_{\Omega}\left|u^{(m)}\right|^{2}+\left|v^{(m)}\right|^{2} \mathrm{~d} x\right) \mathrm{d} t & \leq C \int_{Q_{\tau}}\left|u^{(m)}\right|^{q(\cdot)-1}\left|v^{(m)}\right|+1 \mathrm{~d} z \\
& \leq C \int_{Q_{\tau}}\left|u^{(m)}\right|^{2(q(\cdot)-1)}+\left|v^{(m)}\right|^{2}+1 \mathrm{~d} z \\
& \leq C_{1} \int_{Q_{\tau}}\left|u^{(m)}\right|^{2}+\left|v^{(m)}\right|^{2} \mathrm{~d} z+C_{2}\left|Q_{\tau}\right|,
\end{aligned}
$$

provided $2(q(\cdot)-1) \leq 2$, i.e. $1<q(\cdot) \leq 2$, with constants

$$
C_{1}:= \begin{cases}2 \beta \cdot \max \left\{1,1 / \bar{c}_{*}\right\}, & \text { if } p(\cdot) \equiv 2 \text { and } \bar{c}_{*}:=\left(a_{0}-a_{1}+\min \left\{d_{1} \mu_{1}, d_{2} \mu_{2}\right\}\right) \geq 0, \\ 2 \beta \cdot \bar{c}_{\ddagger}, & \text { if } p(\cdot) \geq \gamma_{1}>2\end{cases}
$$

and

$$
C_{2}:= \begin{cases}0, & \text { if } p(\cdot) \equiv 2 \text { and } \bar{c}_{*}:=\left(a_{0}-a_{1}+\min \left\{d_{1} \mu_{1}, d_{2} \mu_{2}\right\}\right) \geq 0, \\ 2 \beta \cdot \max \left\{1, \bar{c}_{\dagger}\right\}, & \text { if } p(\cdot) \geq \gamma_{1}>2 .\end{cases}
$$

Using Gronwall's inequality, we finally can conclude that 


$$
\begin{aligned}
\sup _{0 \leq \tau \leq T_{m}} \int_{\Omega}\left|u^{(m)}(\cdot, \tau)\right|^{2}+\left|v^{(m)}(\cdot, \tau)\right|^{2} \mathrm{~d} x & +\int_{Q_{\tau}}\left|\nabla u^{(m)}\right|^{2}+\left|\nabla v^{(m)}\right|^{2} \mathrm{~d} z \\
& +\int_{Q_{\tau}}\left|\nabla u^{(m)}\right|^{p(\cdot)}+\left|\nabla v^{(m)}\right|^{p(\cdot)} \mathrm{d} z \\
\leq & T_{m} \exp \left(C_{1} T_{m}\right) \int_{\Omega}\left|u_{0}(x)\right|^{2}+\left|v_{0}(x)\right|^{2} \mathrm{~d} x+T_{m} C_{2}\left|Q_{\tau}\right|
\end{aligned}
$$

for a.e. $\tau \in\left(0, T_{m}\right)$, cf. [7, 9]. Thus, we can extend $\left(0, T_{m}\right)$ to $(0, T)$, which yields

$$
\begin{aligned}
\sup _{0 \leq t \leq T} \int_{\Omega}\left|u^{(m)}(\cdot, t)\right|^{2}+\left|v^{(m)}(\cdot, \tau)\right|^{2} \mathrm{~d} x & +\int_{Q_{T}}\left|\nabla u^{(m)}\right|^{2}+\left|\nabla v^{(m)}\right|^{2} \mathrm{~d} z \\
& +\int_{Q_{T}}\left|\nabla u^{(m)}\right|^{p(x, t)}+\left|\nabla v^{(m)}\right|^{p(x, t)} \mathrm{d} z \\
\leq & \exp \left(C_{1} T\right) \int_{\Omega}\left|u_{0}(x)\right|^{2}+\left|v_{0}(x)\right|^{2} \mathrm{~d} x+T C_{2}\left|Q_{T}\right| \leq c
\end{aligned}
$$

with a constant $c=c\left(a_{0}, a_{1}, d_{1}, d_{2}, \beta, \mu_{1}, \mu_{2}, \gamma_{1}, \gamma_{2},\left\|u_{0}\right\|_{L^{2}},\left\|v_{0}\right\|_{L^{2}},\left|Q_{T}\right|\right)$, cf. [25]. This together with Lemma 1.2, we have that $u^{(m)}$ and $v^{(m)}$ are uniformly bounded in $L^{\infty}\left(0, T ; L^{2}(\Omega)\right) \cap W^{p(\cdot)}\left(Q_{T}\right)$, provided $u_{\Omega}^{(m)}=v_{\Omega}^{(m)}=0$, which implies the weak convergences for the sequences $\left\{u^{(m)}\right\}$ and $\left\{v^{(m)}\right\}$ up to a subsequence

$$
\left\{\begin{array}{l}
u^{(m)} \rightarrow^{*} u \text { and } v^{(m)} \rightarrow^{*} v \text { weakly* in } L^{\infty}\left(0, T ; L^{2}(\Omega)\right), \\
\nabla u^{(m)}-\nabla u \text { and } \nabla v^{(m)} \rightarrow \nabla v \text { weakly in } L^{p(\cdot)}\left(Q_{T}, \mathbb{R}^{n}\right) .
\end{array}\right.
$$

To be able to prove the strong convergence

$$
u^{(m)} \rightarrow u \quad \text { and } \quad v^{(m)} \rightarrow v \quad \text { strongly in } L^{p(\cdot)}\left(Q_{T}\right)
$$

using Theorem 1.4, we first have to prove that

$$
\partial_{t} u^{(m)} \rightarrow \partial_{t} u \text { and } \partial_{t} v^{(m)} \rightarrow \partial_{t} v \text { weakly in } W^{p(\cdot)}\left(Q_{T}\right)^{\prime} .
$$

To this end, we define a subspace of the set of admissible test functions

$$
\mathcal{W}_{m}\left(Q_{T}\right):=\left\{\eta: \eta=\sum_{i=1}^{m} \psi_{i} w_{i}, \psi_{i} \in C^{1}([0, T])\right\} \subset W^{p(\cdot)}\left(Q_{T}\right),
$$

since $W_{0}^{p(\cdot)}\left(Q_{T}\right) \subset W^{p(\cdot)}\left(Q_{T}\right)$. Then, we choose test functions

$$
\varphi(z)=\sum_{i=1}^{m} \psi_{i}(t) w_{i}(x) \in \mathcal{W}_{m}\left(Q_{T}\right), \quad \tilde{\varphi}(z)=\sum_{i=1}^{m} \tilde{\psi}_{i}(t) w_{i}(x) \in \mathcal{W}_{m}\left(Q_{T}\right) .
$$

Note that $\partial_{t} \varphi$ and $\partial_{t} \tilde{\varphi}$ exist, since the coefficients $\psi_{i}(t)$ and $\tilde{\psi}_{i}(t)$ lie in $C^{1}([0, T])$. Thus, we have 


$$
\begin{array}{r}
\int_{Q_{T}} u_{t}^{(m)} \varphi \mathrm{d} z=-\int_{Q_{T}} u^{(m)} \varphi_{t} \mathrm{~d} z+\left.\int_{\Omega} u^{(m)} \varphi \mathrm{d} x\right|_{0} ^{T}=-\int_{Q_{T}} d_{1} A_{1}\left(x, t, \nabla u^{(m)}\right) \cdot \nabla \varphi \mathrm{d} z \\
-\int_{Q_{T}}\left[\alpha_{1}(x, t) \nabla u^{(m)}+\alpha_{2}(x, t) \nabla v^{(m)}\right] \cdot \nabla \varphi \mathrm{d} z
\end{array}
$$

and

$$
\begin{aligned}
\int_{Q_{T}} v_{t}^{(m)} \tilde{\varphi} \mathrm{d} z= & -\int_{Q_{T}} v^{(m)} \tilde{\varphi}_{t} \mathrm{~d} z+\left.\int_{\Omega} v^{(m)} \tilde{\varphi} \mathrm{d} x\right|_{0} ^{T}=-\int_{Q_{T}} d_{2} A_{2}\left(x, t, \nabla v^{(m)}\right) \cdot \nabla \tilde{\varphi} \mathrm{d} z \\
& -\int_{Q_{T}}\left[\alpha_{3}(x, t) \nabla u^{(m)}+\alpha_{4}(x, t) \nabla v^{(m)}\right] \cdot \nabla \tilde{\varphi} \mathrm{d} z \\
& +\beta \int_{Q_{T}}\left|u^{(m)}\right|^{q(\cdot)-2} u^{(m)} \tilde{\varphi} \mathrm{d} z .
\end{aligned}
$$

From this we can conclude that

$$
\begin{aligned}
\left|\int_{Q_{T}} u_{t}^{(m)} \varphi \mathrm{d} z\right| \leq & \int_{Q_{T}}\left|d_{1} A_{1}\left(x, t, \nabla u^{(m)}\right)\right| \cdot(\varphi+|\nabla \varphi|) \mathrm{d} z \\
& +\int_{Q_{T}}\left|\alpha_{1}(x, t) \nabla u^{(m)}+\alpha_{2}(x, t) \nabla v^{(m)}\right| \cdot(\varphi+|\nabla \varphi|) \mathrm{d} z \\
\leq & \int_{Q_{T}}\left(d_{1} L_{1}\left(h_{1}+\left|\nabla u^{(m)}\right|^{p(\cdot)-1}\right)+a_{1}\left(\left|\nabla u^{(m)}\right|+\left|\nabla v^{(m)}\right|\right)\right) \cdot(\varphi+|\nabla \varphi|) \mathrm{d} z \\
\leq & \left(\frac{1}{\gamma_{1}}+\frac{\gamma_{2}-1}{\gamma_{2}}\right)\left\|d_{1} L_{1}\left(h_{1}+\left|\nabla u^{(m)}\right|^{p(\cdot)-1}\right)+a_{1}\left(\left|\nabla u^{(m)}\right|+\left|\nabla v^{(m)}\right|\right)\right\|_{L^{p^{(x, t)}}\left(Q_{T}\right)} \\
& \times\|\varphi\|_{W^{p(\cdot)}\left(Q_{T}\right)} \leq c_{1}\|\varphi\|_{W^{p(\cdot)}\left(Q_{T}\right)}
\end{aligned}
$$

with a constant $c_{1}=c_{1}\left(a_{0}, a_{1}, d_{1}, d_{2}, \mu_{1}, \mu_{2}, \gamma_{1}, \gamma_{2},\left\|u_{0}\right\|_{L^{2}},\left\|v_{0}\right\|_{L^{2}},\left|Q_{T}\right|, L_{1},\left\|h_{1}\right\|_{L^{p^{(\cdot)}}}\right)$, where we applied the generalised Hölder's inequality (1.11), the growth condition (1.5), the condition (1.7), (1.12), the fact $p^{\prime}(x, t) \leq 2 \leq p(x, t)$ and the energy estimate (3.5). Similarly, we can deduce that

$$
\left|\int_{Q_{T}} v_{t}^{(m)} \tilde{\varphi} \mathrm{d} z\right| \leq c_{2}\|\tilde{\varphi}\|_{W^{p(\cdot)}\left(Q_{T}\right)}
$$

with a constant

$$
c_{2}=c_{2}\left(a_{0}, a_{1}, d_{1}, d_{2}, \beta, \mu_{1}, \mu_{2}, \gamma_{1}, \gamma_{2},\left\|u_{0}\right\|_{L^{2}},\left\|v_{0}\right\|_{L^{2}},\left|Q_{T}\right|, L_{2},\left\|h_{2}\right\|_{L^{p^{(}(\cdot)}}, \operatorname{diam}(\Omega), \omega(\cdot)\right),
$$

where we also used the Poincaré type estimate (1.17) with $u_{\Omega}^{(m)}=v_{\Omega}^{(m)}=0$. This shows that

$$
u_{t}^{(m)} \in W^{p(\cdot)}\left(Q_{T}\right)^{\prime} \quad \text { and } \quad v_{t}^{(m)} \in W^{p(\cdot)}\left(Q_{T}\right)^{\prime}
$$

with $\left\|u_{t}^{(m)}\right\|_{W^{p(\cdot)}\left(Q_{T}\right)^{\prime}} \leq c_{1}$ and $\left\|v_{t}^{(m)}\right\|_{W^{p(\cdot)}\left(Q_{T}\right)^{\prime}} \leq c_{2}$. Summarising, we have the weak convergences for the sequences $\left\{u^{(m)}\right\}$ and $\left\{v^{(m)}\right\}$ (up to a subsequence): 


$$
\left\{\begin{array}{l}
u^{(m)} \vec{*}^{*} u \text { and } v^{(m)} \vec{*}^{*} v \text { weakly* in } L^{\infty}\left(0, T ; L^{2}(\Omega)\right), \\
\nabla u^{(m)}-\nabla u \text { and } \nabla v^{(m)} \rightarrow \nabla v \text { weakly in } L^{p(\cdot)}\left(Q_{T}, \mathbb{R}^{n}\right), \\
u_{t}^{(m)} \rightarrow u_{t} \text { and } v_{t}^{(m)} \rightarrow v_{t} \text { weakly in } W^{p(\cdot)}\left(Q_{T}\right)^{\prime}
\end{array}\right.
$$

provided $u_{\Omega}^{(m)}=v_{\Omega}^{(m)}=0$. Moreover, by Theorem 1.4 we can conclude that the sequences $\left\{u^{(m)}\right\}$ and $\left\{v^{(m)}\right\}$ (up to a subsequence) converges strongly in $L^{p(\cdot)}\left(Q_{T}\right)$ to some function $u, v \in W\left(Q_{T}\right)$ with $u_{\Omega}=v_{\Omega}=0$. Thus, we have the desired convergences

$$
\left\{\begin{array}{l}
u^{(m)} \rightarrow u \text { and } v^{(m)} \rightarrow v \text { strongly in } L^{p(\cdot)}\left(Q_{T}\right), \\
u^{(m)} \rightarrow u \text { and } v^{(m)} \rightarrow v \text { a.e. in } Q_{T} .
\end{array}\right.
$$

In addition, the growth assumption on $A_{i}(z, \cdot)$ and the estimate (3.5) imply that the sequences $\left\{A_{1}\left(z, \nabla u^{(m)}\right)\right\}_{m \in \mathbb{N}}$ and $\left\{A_{2}\left(z, \nabla v^{(m)}\right)\right\}_{m \in \mathbb{N}}$ are bounded in $L^{p^{\prime}(\cdot)}\left(Q_{T}, \mathbb{R}^{n}\right)$. Consequently, after passing to a subsequence once more, we can find limit maps $A_{1_{0}}, A_{2_{0}} \in L^{p^{\prime}(\cdot)}\left(Q_{T}, \mathbb{R}^{n}\right)$ with

$$
\begin{aligned}
& A_{1}\left(z, \nabla u^{(m)}\right) \rightarrow A_{1_{0}} \quad \text { as } m \rightarrow \infty, \\
& A_{2}\left(z, \nabla v^{(m)}\right) \rightarrow A_{2_{0}} \quad \text { as } m \rightarrow \infty .
\end{aligned}
$$

The next aim is to show that

$$
A_{1_{0}}=A_{1}(x, t, \nabla u) \quad \text { and } \quad A_{2_{0}}=A_{2}(x, t, \nabla v) \quad \text { for almost every }(x, t) \in Q_{T} .
$$

By the method of construction [7], we have from (3.2) and (3.3) for all test function $\phi_{i} \in \mathcal{W}_{s}\left(Q_{T}\right), i=1,2$ with $s \leq m$ for an arbitrary fixed $m \in \mathbb{N}$ that

$$
-\int_{Q_{T}} u_{t}^{(m)} \phi_{1}+\left[d_{1} A_{1}\left(x, t, \nabla u^{(m)}\right)+\alpha_{1}(x, t) \nabla u^{(m)}+\alpha_{2}(x, t) \nabla v^{(m)}\right] \nabla \phi_{1} \mathrm{~d} z=0
$$

and

$$
\begin{aligned}
-\int_{Q_{T}} v_{t}^{(m)} \phi_{2} & +\left[d_{2} A_{2}\left(x, t, \nabla v^{(m)}\right)+\alpha_{3}(x, t) \nabla u^{(m)}+\alpha_{4}(x, t) \nabla v^{(m)}\right] \nabla \phi_{2} \mathrm{~d} z \\
& =\beta \int_{Q_{T}}\left|u^{(m)}\right|^{q(\cdot)-2} u^{(m)} \cdot \phi_{2} \mathrm{~d} z .
\end{aligned}
$$

Then, passing to the limit $m \rightarrow \infty$ we get

$$
-\int_{Q_{T}} u_{t} \phi_{1}+\left[d_{1} A_{1_{0}}+\alpha_{1}(x, t) \nabla u+\alpha_{2}(x, t) \nabla v\right] \nabla \phi_{1} \mathrm{~d} z=0
$$

and 


$$
-\int_{Q_{T}} v_{t} \phi_{2}+\left[d_{2} A_{2_{0}}+\alpha_{3}(x, t) \nabla u+\alpha_{4}(x, t) \nabla v\right] \nabla \phi_{2} \mathrm{~d} z=\beta \int_{Q_{T}}|u|^{q(\cdot)-2} u \cdot \phi_{2} \mathrm{~d} z
$$

for every $\phi_{i} \in \mathcal{W}_{s}\left(Q_{T}\right), i=1,2$. According to the monotonicity assumption (1.6), we also know that

$$
\begin{aligned}
& \int_{Q_{T}} d_{1}\left(A_{1}\left(x, t, \nabla u^{(m)}\right)-A_{1}\left(x, t, \nabla \xi_{1}\right)\right) \cdot \nabla\left(u^{(m)}-\xi_{1}\right) \mathrm{d} z \geq 0 \\
& \int_{Q_{T}} d_{2}\left(A_{2}\left(x, t, \nabla v^{(m)}\right)-A_{2}\left(x, t, \nabla \xi_{2}\right)\right) \cdot \nabla\left(v^{(m)}-\xi_{2}\right) \mathrm{d} z \geq 0
\end{aligned}
$$

for every $\xi_{i} \in \mathcal{W}_{s}\left(Q_{T}\right), i=1,2$. Choosing $\phi_{1}=\left(u^{(m)}-\xi_{1}\right)$ and $\phi_{2}=\left(v^{(m)}-\xi_{2}\right)$ with $\xi_{i} \in \mathcal{W}_{s}\left(Q_{T}\right), i=1,2$ as admissible test functions, we can conclude from (3.7), (3.8) and (3.11) that

$$
\begin{array}{r}
-\int_{Q_{T}} u_{t}^{(m)} \phi_{1}+\left[d_{1} A_{1}\left(x, t, \nabla u^{(m)}\right)+\alpha_{1}(x, t) \nabla u^{(m)}+\alpha_{2}(x, t) \nabla v^{(m)}\right] \nabla \phi_{1} \mathrm{~d} z \\
+\int_{Q_{T}} d_{1}\left(A_{1}\left(x, t, \nabla u^{(m)}\right)-A_{1}\left(x, t, \nabla \xi_{1}\right)\right) \cdot \nabla \phi_{1} \mathrm{~d} z \geq 0
\end{array}
$$

and

$$
\begin{array}{r}
-\int_{Q_{T}} v_{t}^{(m)} \phi_{2}+\left[d_{2} A_{2}\left(x, t, \nabla v^{(m)}\right)+\alpha_{3}(x, t) \nabla u^{(m)}+\alpha_{4}(x, t) \nabla v^{(m)}\right] \nabla \phi_{2} \mathrm{~d} z \\
-\beta \int_{Q_{T}}\left|u^{(m)}\right|^{q(x, t)-2} u^{(m)} \cdot \phi_{2} \mathrm{~d} z+\int_{Q_{T}} d_{2}\left(A_{2}\left(x, t, \nabla v^{(m)}\right)-A_{2}\left(x, t, \nabla \xi_{2}\right)\right) \cdot \nabla \phi_{2} \mathrm{~d} z \geq 0,
\end{array}
$$

which implies

$$
-\int_{Q_{T}} u_{t}^{(m)} \phi_{1}+\left[d_{1} A_{1}\left(x, t, \nabla \xi_{1}\right)+\alpha_{1}(x, t) \nabla u^{(m)}+\alpha_{2}(x, t) \nabla v^{(m)}\right] \nabla \phi_{1} \mathrm{~d} z \geq 0
$$

and

$$
\begin{aligned}
-\int_{Q_{T}} v_{t}^{(m)} \phi_{2} & +\left[d_{2} A_{2}\left(x, t, \nabla \xi_{2}\right)+\alpha_{3}(x, t) \nabla u^{(m)}+\alpha_{4}(x, t) \nabla v^{(m)}\right] \nabla \phi_{2} \mathrm{~d} z \\
& -\beta \int_{Q_{T}}\left|u^{(m)}\right|^{q(\cdot)-2} u^{(m)} \cdot \phi_{2} \mathrm{~d} z \geq 0
\end{aligned}
$$

where $\phi_{1}=\left(u^{(m)}-\xi_{1}\right)$ and $\phi_{2}=\left(v^{(m)}-\xi_{2}\right)$ with $\xi_{i} \in \mathcal{W}_{s}\left(Q_{T}\right), i=1,2$. Next, testing (3.9) and (3.10) with $\phi_{1}=\left(u^{(m)}-\xi_{1}\right)$ and $\phi_{2}=\left(v^{(m)}-\xi_{2}\right)$ with $\xi_{i} \in \mathcal{W}_{s}\left(Q_{T}\right), i=1,2$ we can deduce by subtracting (3.9) and (3.10) from (3.12) and (3.13), respectively, and passing to the limit $m \rightarrow \infty$ that 


$$
\begin{gathered}
-\int_{Q_{T}} d_{1}\left(A_{1}\left(x, t, \nabla \xi_{1}\right)-A_{1_{0}}\right) \nabla\left(u-\xi_{1}\right) \mathrm{d} z \geq 0, \\
-\int_{Q_{T}} d_{2}\left(A_{2}\left(x, t, \nabla \xi_{2}\right)-A_{2_{0}}\right) \nabla\left(v-\xi_{2}\right) \mathrm{d} z \geq 0
\end{gathered}
$$

for all $\xi_{i} \in \mathcal{W}_{s}\left(Q_{T}\right), i=1,2$. Then, choosing $\xi_{1}=u \pm \varepsilon \psi_{1}$ and $\xi_{2}=v \pm \varepsilon \psi_{2}$ with arbitrary $\psi_{i} \in W^{p(\cdot)}\left(Q_{T}\right)$ finally yields

$$
\begin{aligned}
& -\varepsilon \int_{Q_{T}} d_{1}\left(A_{1}\left(x, t, \nabla\left(u \pm \varepsilon \psi_{1}\right)\right)-A_{1_{0}}\right) \nabla \psi_{1} \mathrm{~d} z \geq 0, \\
& -\varepsilon \int_{Q_{T}} d_{2}\left(A_{2}\left(x, t, \nabla\left(u \pm \varepsilon \psi_{2}\right)\right)-A_{2_{0}}\right) \nabla \psi_{2} \mathrm{~d} z \geq 0 .
\end{aligned}
$$

Passing to the limit $\varepsilon \downarrow 0$, then implies

$$
A_{1_{0}}=A_{1}(x, t, \nabla u) \quad \text { and } \quad A_{2_{0}}=A_{2}(x, t, \nabla v) \quad \text { for almost every }(x, t) \in Q_{T} .
$$

The last step in our existence proof is to check if the initial value condition is satisfied, which is similar to $[9,13]$. Consider functions

$$
\phi_{i}(x, t)=\sum_{k=1}^{m} \psi_{k}^{i}(t) w_{k}(x) \quad i=1,2,
$$

where $\psi_{k}^{i} \in C^{1}([0, T])$. Then, choose test functions from (3.14) with $\phi_{i}(\cdot, T)=0$. Thus, we can conclude from (3.7) and (3.8), integrating by parts and passing to the limit $m \rightarrow \infty$ the following:

$$
\int_{Q_{T}} u \phi_{1 t}-d_{1} A_{1}(x, t, \nabla u) \cdot \nabla \phi_{1}-\left[\alpha_{1}(x, t) \nabla u+\alpha_{2}(x, t) \nabla v\right] \cdot \nabla \phi_{1} \mathrm{~d} z=\int_{\Omega} u_{0} \cdot \phi_{1}(x, 0) \mathrm{d} x
$$

where we used $u^{(m)}(\cdot, 0) \rightarrow u_{0}$ as $m \rightarrow \infty$, cf. [25], and similarly

$$
\begin{aligned}
\int_{Q_{T}} v \phi_{2 t}-d_{2} A_{2}(x, t, \nabla v) \cdot \nabla \phi_{2}-\left[\alpha_{3}(x, t) \nabla u+\alpha_{4}(x, t) \nabla v\right] \cdot \nabla \phi_{2} \mathrm{~d} z & =\int_{\Omega} v_{0} \cdot \phi_{2}(x, 0) \mathrm{d} x \\
& -\beta \int_{Q_{T}}|u|^{q(\cdot)-2} u \phi_{2} \mathrm{~d} z .
\end{aligned}
$$

On the other side hand we know from (3.9) and (3.10) that

$$
\int_{Q_{T}} u \phi_{1 t}-d_{1} A_{1}(x, t, \nabla u) \cdot \nabla \phi_{1}-\left[\alpha_{1}(x, t) \nabla u+\alpha_{2}(x, t) \nabla v\right] \cdot \nabla \phi_{1} \mathrm{~d} z=\int_{\Omega}\left(u \cdot \phi_{1}\right)(x, 0) \mathrm{d} x
$$

and 


$$
\begin{aligned}
\int_{Q_{T}} v \phi_{2 t}-d_{2} A_{2}(x, t, \nabla v) \cdot \nabla \phi_{2}-\left[\alpha_{3}(x, t) \nabla u+\alpha_{4}(x, t) \nabla v\right] \cdot \nabla \phi_{2} \mathrm{~d} z & =\int_{\Omega}\left(v \cdot \phi_{2}\right)(x, 0) \mathrm{d} x \\
& -\beta \int_{Q_{T}}|u|^{q(\cdot)-2} u \phi_{2} \mathrm{~d} z .
\end{aligned}
$$

Finally, since $\phi_{i}$ are arbitrary we have that $u(\cdot, 0)=u_{0}$ and $v(\cdot, 0)=v_{0}$, which completes the proof.

Remark 3.1 There are some additional assumptions one can make to weaken the condition on the exponent $q(x, t)$ :

i) For a modified version of problem (1.1), i.e.

$$
\left\{\begin{array}{l}
\partial_{t} u=d_{1} \operatorname{div}\left(A_{1}(x, t, \nabla u)\right)+\operatorname{div}\left(\alpha_{1}(x, t) \nabla u+\alpha_{2}(x, t) \nabla v\right)-|u|^{q(x, t)-2} u+|v|^{q(x, t)-2} v,(x, t) \in Q_{T} \\
\partial_{t} v=d_{2} \operatorname{div}\left(A_{2}(x, t, \nabla v)\right)+\operatorname{div}\left(\alpha_{3}(x, t) \nabla u+\alpha_{4}(x, t) \nabla v\right)+|u|^{q(x, t)-2} u-|v|^{q(x, t)-2} v,(x, t) \in Q_{T} \\
\frac{\partial u}{\partial v}=\frac{\partial v}{\partial v}=0, \quad(x, t) \in S_{T} \\
u(x, 0)=u_{0}(x), v(x, 0)=v_{0}(x), \quad x \in \Omega,
\end{array}\right.
$$

we do not need Gronwall's inequality to derive the energy estimate (3.5), since we would have

$$
-\int_{Q_{\tau}}\left(\left|u^{(m)}\right|^{q(x, t)-2} u^{(m)}-\left|v^{(m)}\right|^{q(x, t)-2} v^{(m)}\right)\left(u^{(m)}-v^{(m)}\right) \mathrm{d} z \leq 0
$$

for any $1<q(x, t)<\infty$, cf. Lemma 1.6, instead of

$$
\int_{Q_{\tau}}\left|u^{(m)}\right|^{q(x, t)-2} u^{(m)} v^{(m)} \mathrm{d} z .
$$

Thus, we don't need the restriction $1<q(x, t) \leq 2$.

ii) An other approach would be the following: Assume that there exist constants $q^{-}$ and $q^{+}$, such that $1<q^{-} \leq q(x, t) \leq q^{+} \leq p(x, t)$, then we can conclude that

$$
\begin{aligned}
\int_{Q_{\tau}}\left|u^{(m)}\right|^{q(x, t)-2} u^{(m)} v^{(m)} \mathrm{d} z & \leq\left(\int_{Q_{\tau}}\left|u^{(m)}\right|^{\frac{q(x, t)-1}{q^{+}-1} q^{+}} \mathrm{d} z\right)^{\frac{q^{+}-1}{q^{+}}}\left(\int_{Q_{\tau}}\left|v^{(m)}\right|^{q^{+}} \mathrm{d} z\right)^{\frac{1}{q^{+}}} \\
& \leq\left(c_{1} \int_{Q_{\tau}}\left|\nabla u^{(m)}\right|^{q^{+}}+1 \mathrm{~d} z\right)^{\frac{q^{+}-1}{q^{+}}}\left(c_{2} \int_{Q_{\tau}}\left|\nabla v^{(m)}\right|^{q^{+}} \mathrm{d} z\right)^{\frac{1}{q^{+}}} \\
& \leq \frac{q^{+}-1}{q^{+}} c_{1} \int_{Q_{\tau}}\left|\nabla u^{(m)}\right|^{p(x, t)} \mathrm{d} z+\frac{c_{2}}{q^{+}} \int_{Q_{\tau}}\left|\nabla v^{(m)}\right|^{p(x, t)} \mathrm{d} z+c_{3}\left|Q_{\tau}\right|
\end{aligned}
$$

with constants $c_{1}, c_{2}$ and $c_{3}$ dependent on $\left(n, q^{-}, q^{+}\right.$, $\left.\operatorname{diam}(\Omega), \gamma_{1}, \gamma_{2}\right)$, where we used Hölder's, Poincaré's and Young's inequality to derive this estimate. To be able to absorb these terms on the left-hand side the structure constants of system (1.1) have to satisfy 


$$
\begin{cases}\bar{c}_{*} \geq \max \left\{\frac{q^{+}-1}{q^{+}} c_{1}, \frac{c_{2}}{q^{+}}\right\}, & \text {if } p(\cdot) \equiv 2 \text { and } \bar{c}_{*}:=\left(a_{0}-a_{1}+\min \left\{d_{1} \mu_{1}, d_{2} \mu_{2}\right\}\right) \geq 0, \\ 1 \geq \bar{c}_{\ddagger} \cdot \max \left\{\frac{q^{+}-1}{q^{+}} c_{1}, \frac{c_{2}}{q^{+}}\right\}, & \text {if } p(\cdot) \geq \gamma_{1}>2,\end{cases}
$$

where

$$
\bar{c}_{\ddagger}:=\frac{2 \gamma_{1}}{\gamma_{1}-1} \max \left\{1, \frac{1}{\min \left\{a_{0}, d_{1} \mu_{1}, d_{2} \mu_{2}\right\}}\right\} .
$$

Thus, we don't need again the restriction $1<q(x, t) \leq 2$, but other restrictions on the system coefficients.

\section{Proof of the uniqueness result}

Now, we are in the situation to prove the uniqueness of the weak solution to problem (1.1) according to Theorem 2.2.

Proof of Theorem 2.2 For the proof of uniqueness, we assume that there exist two pairs of solutions $(u, v)$ and $\left(u_{1}, v_{1}\right)$ with the same initial value $\left(u_{0}, v_{0}\right)$. Therefore, we choose $\phi_{1}=u-u_{1}$ and $\phi_{2}=v-v_{1}$ as admissible test functions.

i) Subtracting the weak formulation for $\left(u_{1}, v_{1}\right)$ from the weak formulation for $(u, v)$, [cf. (1.14) \& (1.15)] and using integration by parts, we get

$$
\begin{aligned}
0=\int_{Q_{T}}\left(u-u_{1}\right)_{t}\left(u-u_{1}\right) & +d_{1}\left(A_{1}(x, t, \nabla u)-A_{1}\left(x, t, \nabla u_{1}\right)\right) \nabla\left(u-u_{1}\right) \mathrm{d} z \\
& +\left(\alpha_{1}(x, t) \nabla\left(u-u_{1}\right)+\alpha_{2}(x, t) \nabla\left(v-v_{1}\right)\right) \nabla\left(u-u_{1}\right) \mathrm{d} z \\
\geq \int_{Q_{T}}\left(u-u_{1}\right)_{t}\left(u-u_{1}\right) & +\alpha_{2}(x, t) \nabla\left(v-v_{1}\right) \nabla\left(u-u_{1}\right)+\left(a_{0}+d_{1} \mu_{1}\right)\left|\nabla\left(u-u_{1}\right)\right|^{2} \mathrm{~d} z,
\end{aligned}
$$

where we applied the monotonicity condition (2.2) and (1.7). Similarly, we have

$$
\begin{aligned}
0=\int_{Q_{T}}\left(v-v_{1}\right)_{t}\left(v-v_{1}\right) & +d_{2}\left(A_{2}(x, t, \nabla v)-A_{2}\left(x, t, \nabla v_{1}\right)\right) \nabla\left(v-v_{1}\right) \mathrm{d} z \\
& +\left(\alpha_{3}(x, t) \nabla\left(u-u_{1}\right)+\alpha_{4}(x, t) \nabla\left(v-v_{1}\right)\right) \nabla\left(v-v_{1}\right) \mathrm{d} z \\
& +\int_{Q_{T}}\left(|u|^{q(\cdot)-2} u-\left|u_{1}\right|^{q(\cdot)-2} u_{1}\right)\left(v-v_{1}\right) \mathrm{d} z \\
\geq \int_{Q_{T}}\left(v-v_{1}\right)_{t}\left(v-v_{1}\right) & +\alpha_{3}(x, t) \nabla\left(u-u_{1}\right) \nabla\left(v-v_{1}\right)+\left(a_{0}+d_{2} \mu_{2}\right)\left|\nabla\left(v-v_{1}\right)\right|^{2} \mathrm{~d} z \\
& +\beta \int_{Q_{T}}\left(|u|^{q(\cdot)-2} u-\left|u_{1}\right|^{q(\cdot)-2} u_{1}\right)\left(v-v_{1}\right) \mathrm{d} z .
\end{aligned}
$$

Combining these estimates, using Cauchy's and Hölder's inequality, we get 


$$
\begin{aligned}
& \beta\left(\left.\int_{Q_{T}}|| u\right|^{q(\cdot)-2} u-\left.\left|u_{1}\right|^{q(\cdot)-2} u_{1}\right|^{2} \mathrm{~d} z\right)^{\frac{1}{2}}\left(\int_{Q_{T}}\left|v-v_{1}\right|^{2} \mathrm{~d} z\right)^{\frac{1}{2}} \\
& \geq \int_{Q_{T}}\left(u-u_{1}\right)_{t}\left(u-u_{1}\right)+\left(v-v_{1}\right)_{t}\left(v-v_{1}\right) \\
& \quad+\left(\alpha_{2}(x, t)+\alpha_{3}(x, t)\right) \nabla\left(v-v_{1}\right) \nabla\left(u-u_{1}\right) \mathrm{d} z \\
& \quad+\left(a_{0}+\min \left\{d_{1} \mu_{1}, d_{2} \mu_{2}\right\}\right) \int_{Q_{T}}\left|\nabla\left(u-u_{1}\right)\right|^{2}+\left|\nabla\left(v-v_{1}\right)\right|^{2} \mathrm{~d} z .
\end{aligned}
$$

Using again Cauchy's inequality and (1.7) we further can conclude that

$$
\begin{aligned}
& \beta\left(\left.\int_{Q_{T}}|| u\right|^{q(\cdot)-2} u-\left.\left|u_{1}\right|^{q(\cdot)-2} u_{1}\right|^{2} \mathrm{~d} z\right)^{\frac{1}{2}}\left(\int_{Q_{T}}\left|v-v_{1}\right|^{2} \mathrm{~d} z\right)^{\frac{1}{2}} \mathrm{~d} z \\
& \geq \frac{1}{2} \int_{0}^{T}\left(\frac{\mathrm{d}}{\mathrm{d} t} \int_{\Omega}\left|u-u_{1}\right|^{2}+\left|v-v_{1}\right|^{2} \mathrm{~d} x\right) \mathrm{d} t \\
& \quad+\left(a_{0}-a_{1}+\min \left\{d_{1} \mu_{1}, d_{2} \mu_{2}\right\}\right) \int_{Q_{T}}\left|\nabla\left(u-u_{1}\right)\right|^{2}+\left|\nabla\left(v-v_{1}\right)\right|^{2} \mathrm{~d} z .
\end{aligned}
$$

For $q(\cdot) \equiv 2$ for all $\beta>0$ and under the assumption (2.3), we can immediately conclude that

$$
\int_{0}^{T} \frac{\mathrm{d}}{\mathrm{d} t}\left(\left\|v-v_{1}\right\|_{L^{2}(\Omega)}^{2}+\left\|u-u_{1}\right\|_{L^{2}(\Omega)}^{2}\right) \mathrm{d} t \leq \beta \int_{0}^{T}\left\|v-v_{1}\right\|_{L^{2}(\Omega)}^{2}+\left\|u-u_{1}\right\|_{L^{2}(\Omega)}^{2} \mathrm{~d} t
$$

and by means of Gronwall's inequality (differential form) we gain

$$
0 \leq\left\|u-u_{1}\right\|_{L^{2}(\Omega)}^{2}+\left\|v-v_{1}\right\|_{L^{2}(\Omega)}^{2} \leq 0
$$

for every $t \in(0, T)$, since $u(x, 0)-u_{1}(x, 0)=0$ and $v(x, 0)-v_{1}(x, 0)=0$. For $\beta=0$ the uniqueness follows similarly.

The proof of ii) is similar to the proof of i), but we use the monotonicity condition (1.6). Thus, we derive at

$$
\begin{aligned}
\left.\beta \int_{Q_{T}}|| u\right|^{q(\cdot)-2} u-\left|u_{1}\right|^{q(\cdot)-2} u_{1}|| v-v_{1} \mid \mathrm{d} z \geq & \frac{1}{2} \int_{0}^{T}\left(\frac{\mathrm{d}}{\mathrm{d} t} \int_{\Omega}\left|u-u_{1}\right|^{2}+\left|v-v_{1}\right|^{2} \mathrm{~d} x\right) \mathrm{d} t \\
& +\left(a_{0}-a_{1}\right) \int_{Q_{T}}\left|\nabla\left(u-u_{1}\right)\right|^{2}+\left|\nabla\left(v-v_{1}\right)\right|^{2} \mathrm{~d} z
\end{aligned}
$$

which implies similarly the uniqueness (as above) due to the assumption that $a_{0}=\alpha_{k}(x, t)=a_{1}=$ const. Thus, we neither need (2.2) nor (2.3).

Finally, the proof of iii) is now trivial and remains to the reader. This completes the proof.

The proof of the stability estimate of Lemma 2.3 is very similar to the proof of the uniqueness Theorem 2.2. 
Proof of Lemma 2.3 We assume that there exist two pairs of solutions $(u, v)$ and $\left(u_{1}, v_{1}\right)$ with the different initial values $\left(u_{0}, v_{0}\right) \in\left(L^{2}(\Omega)\right)^{2}$ and $\left(u_{1_{0}}, v_{1_{0}}\right) \in\left(L^{2}(\Omega)\right)^{2}$. Then, following the proof of Theorem 2.2, we can conclude for $\beta=0$ that

$$
\frac{1}{2} \int_{0}^{T}\left(\frac{\mathrm{d}}{\mathrm{d} t}\left\|u-u_{1}\right\|_{L^{2}(\Omega)}^{2}+\left\|v-v_{1}\right\|_{L^{2}(\Omega)}^{2}\right) \mathrm{d} t \leq 0
$$

which implies

$$
0 \leq\left\|u-u_{1}\right\|_{L^{2}(\Omega)}^{2}+\left\|v-v_{1}\right\|_{L^{2}(\Omega)}^{2} \leq\left\|u_{0}-u_{1_{0}}\right\|_{L^{2}(\Omega)}^{2}+\left\|v_{0}-v_{1_{0}}\right\|_{L^{2}(\Omega)}^{2}
$$

for a.e. $t \in[0, T)$.

\section{Proof of the nonnegativity of the weak solutions}

Our finally aim is to prove of the nonnegativity of the weak solutions.

Proof of Theorem 2.4 Consider $u^{-}:=\min \{u, 0\}$ and $v^{-}:=\min \{v, 0\}$ with $u(x, 0)=u_{0}(x) \geq 0, v(x, 0)=v_{0}(x) \geq 0, x \in \Omega$. Choosing $\phi_{1}=u^{-}$and $\phi_{2}=v^{-}$in (1.14) and (1.15), integrating over $\Omega$ instead of $\Omega_{T}$ and integration by parts yields

$$
\int_{\Omega} u_{t} \phi_{1}+\left[d_{1} A_{1}(x, t, \nabla u)+\alpha_{1}(x, t) \nabla u+\alpha_{2}(x, t) \nabla v\right] \cdot \nabla \phi_{1} \mathrm{~d} x=0
$$

and

$$
\int_{\Omega} v_{t} \phi_{2}+\left[d_{2} A_{2}(x, t, \nabla v)+\alpha_{3}(x, t) \nabla u+\alpha_{4}(x, t) \nabla v\right] \cdot \nabla \phi_{2}+\beta|u|^{q(x, t)-2} u \phi_{2} \mathrm{~d} x=0 .
$$

Please note that we have $A_{i}(x, t, \xi) \cdot \xi^{-} \geq 0$ either due to the coercivity condition (1.4), since $A_{i}(x, t, \xi) \cdot \xi^{-}=A_{i}(x, t, \xi) \cdot \xi \quad$ or $A_{i}(x, t, \xi) \cdot \xi^{-}=0$, since $\xi^{-}=\min \{\xi, 0\}=0$. Furthermore, we know that

$$
\nabla u \cdot \nabla u^{-}=\nabla u^{-} \cdot \nabla u^{-}=\left|\nabla u^{-}\right|^{2} \text { and } \nabla v \cdot \nabla v^{-}=\nabla v^{-} \cdot \nabla v^{-}=\left|\nabla v^{-}\right|^{2}
$$

due to the fact if $u^{-}=0$ or $v^{-}=0$, then we also have $\nabla u^{-}=0$ or $\nabla v^{-}=0$, respectively and therefore, $\nabla u \cdot \nabla u^{-}=0$ or $\nabla v \cdot \nabla v^{-}=0$. Otherwise, i.e. if $u^{-}=u$ or $v^{-}=v$ we have also $\nabla u \cdot \nabla u^{-}=|\nabla u|^{2}$ or $\nabla v \cdot \nabla v^{-}=|\nabla v|^{2}$. Therefore, we can conclude by means of the coercivity condition (1.4), (1.7) and either (2.4) or (2.5) with (2.6) and the abbreviation $a_{0}=\min \left\{\alpha_{1_{0}}, \alpha_{4_{0}}\right\}$ that 


$$
\begin{aligned}
& \frac{1}{2}\left(\frac{\mathrm{d}}{\mathrm{d} t} \int_{\Omega}\left|u^{-}\right|^{2}+\left|v^{-}\right|^{2} \mathrm{~d} x\right)+a_{0}\left\|\nabla u^{-}\right\|_{L^{2}(\Omega)}^{2}+a_{0}\left\|\nabla v^{-}\right\|_{L^{2}(\Omega)}^{2} \\
& \quad+\min \left\{d_{1} \mu_{1}, d_{2} \mu_{2}\right\} \int_{\Omega}\left|\nabla u^{-}\right|^{p(\cdot)}+\left|\nabla v^{-}\right|^{p(\cdot)} \mathrm{d} x \\
& \leq-\int_{\Omega}\left(\alpha_{2}(x, t) \nabla v \nabla u^{-}+\alpha_{3}(x, t) \nabla v^{-} \nabla u\right) \mathrm{d} x \\
& \quad+\beta \int_{\Omega}\left|u^{-}\right|^{q(\cdot)-1} \cdot v^{-} \mathrm{d} x \\
& \leq a_{0}\left\|\nabla u^{-}\right\|_{L^{2}(\Omega)}^{2}+a_{0}\left\|\nabla v^{-}\right\|_{L^{2}(\Omega)}^{2} \\
& \quad+\beta \int_{\Omega}\left|u^{-}\right|^{q(\cdot)-1} \cdot v^{-} \mathrm{d} x,
\end{aligned}
$$

where we used Hölder's inequality, Young's inequality, the fact that $\nabla u^{-}$is either $\nabla u$ or 0 and $\nabla v^{-}$is either $\nabla v$ or 0 . This implies

$$
\frac{1}{2}\left(\frac{\mathrm{d}}{\mathrm{d} t} \int_{\Omega}\left|u^{-}\right|^{2}+\left|v^{-}\right|^{2} \mathrm{~d} x\right) \leq \beta \int_{\Omega}\left|u^{-}\right|^{q(\cdot)-1} \cdot v^{-} \mathrm{d} x \leq 0 .
$$

for all $t \in[0, T)$. Finally, by the fact that $u^{-}=v^{-}=0$ at $t=0$, since $u(x, 0)=u_{0}(x) \geq 0, v(x, 0)=v_{0}(x) \geq 0$, the last inequality implies that $\left\|u^{-}\right\|_{L^{2}(\Omega)}=\left\|v^{-}\right\|_{L^{2}(\Omega)}=0$ for all $t>0$. Therefore, the solution $(u, v)$ is nonnegative.

Acknowledgements Open Access funding provided by University of Oslo (incl Oslo University Hospital). G.A. is supported by DST/SERB, Grant number EMR/2015/001908. A.E. wishes to thank Erik Wahlén and the Centre of Mathematical Sciences, Lund University, Sweden for hosting him during his research stay in 2020 supported by the Kristine Bonnevie scholarship 2020. The authors wish to thank the anonymous referee for her/his careful reading of the original manuscript and their comments that eventually led to an improved presentation.

\section{Compliance with ethical standards}

Conflict of interest The authors declare that they have no conflict of interest.

Open Access This article is licensed under a Creative Commons Attribution 4.0 International License, which permits use, sharing, adaptation, distribution and reproduction in any medium or format, as long as you give appropriate credit to the original author(s) and the source, provide a link to the Creative Commons licence, and indicate if changes were made. The images or other third party material in this article are included in the article's Creative Commons licence, unless indicated otherwise in a credit line to the material. If material is not included in the article's Creative Commons licence and your intended use is not permitted by statutory regulation or exceeds the permitted use, you will need to obtain permission directly from the copyright holder. To view a copy of this licence, visit http://creativecommons.org/licen ses/by/4.0/. 


\section{References}

1. Aboulaich, R., Meskine, D., Souissi, A.: New diffusion models in image processing. Comput. Math. Appl. 56(4), 874-882 (2008)

2. Acerbi, E., Mingione, G.: Regularity results for electrorheological fluids: the stationary case. C. R. Math. Acad. Sci. Paris 334(9), 817-822 (2002)

3. Acerbi, E., Mingione, G.: Regularity results for stationary electro-rheological fluids. Arch. Ration. Mech. Anal. 164(3), 213-259 (2002)

4. Alkhutov, Y.A., Zhikov, V.V.: Existence theorems for solutions of parabolic equations with a variable order of nonlinearity. Tr. Mat. Inst. Steklova 270, 21-32 (2010)

5. Antontsev, S., Kuznetsov, I., Shmarev, S.: Global higher regularity of solutions to singular $p(x, t)$-parabolic equations. J. Math. Anal. Appl. 466(1), 238-263 (2018)

6. Antontsev, S., Shmarev, S.: A model porous medium equation with variable exponent of nonlinearity: existence, uniqueness and localization properties of solutions. Nonlinear Anal. 60(3), 515-545 (2005)

7. Antontsev, S., Shmarev, S.: Anisotropic parabolic equations with variable nonlinearity. Publ. Math. 53(2), 355-399 (2009)

8. Antontsev, S., Shmarev, S.: Vanishing solutions of anisotropic parabolic equations with variable nonlinearity. J. Math. Anal. Appl. 361(2), 371-391 (2010)

9. Antontsev, S., Shmarev, S.: Evolution PDEs with Nonstandard Growth Conditions. Atlantis Studies in Differential Equations, vol. 4. Atlantis Press, Paris (2015)

10. Antontsev, S., Shmarev, S.: On a class of fully nonlinear parabolic equations. Adv. Nonlinear Anal. 8(1), 79-100 (2019)

11. Antontsev, S., Zhikov, V.: Higher integrability for parabolic equations of $p(x, t)$-Laplacian type. Adv. Differ. Equ. 10(9), 1053-1080 (2005)

12. Arumugam, G., Erhardt, A.H., Eswaramoorthy, I., Krishnan, B.: Existence of weak solutions to the Keller-Segel chemotaxis system with additional cross-diffusion. Nonlinear Anal. Real World Appl. 54, 103090 (2020)

13. Bendahmane, M., Langlais, M.: A reaction-diffusion system with cross-diffusion modeling the spread of an epidemic disease. J. Evol. Equ. 10(4), 883-904 (2010)

14. Bhuvaneswari, V., Lingeshwaran, S., Balachandran, K.: Weak solutions for $p$-Laplacian equation. Adv. Nonlinear Anal. 1(4), 319-334 (2012)

15. Chen, X., Jüngel, A.: A note on the uniqueness of weak solutions to a class of cross-diffusion systems. J. Evol. Equ. 18(2), 805-820 (2018)

16. Chen, Y., Levine, S., Rao, M.: Variable exponent, linear growth functionals in image restoration. SIAM J. Appl. Math. 66(4), 1383-1406 (2006)

17. Chorfi, N., Rădulescu, V.D.: Small perturbations of elliptic problems with variable growth. Appl. Math. Lett. 74, 167-173 (2017)

18. Diening, L., Harjulehto, P., Peter, H., Ružička, M.: Lebesgue and Sobolev Spaces with Variable Exponents. Lecture Notes in Mathematics, vol. 2017. Springer, Heidelberg (2011)

19. Diening, L., Nägele, P., Ružička, M.: Monotone operator theory for unsteady problems in variable exponent spaces. Complex Var. Elliptic Equ. 57(11), 1209-1231 (2012)

20. Eleuteri, M., Harjulehto, P., Lukkari, T.: Global regularity and stability of solutions to obstacle problems with nonstandard growth. Rev. Mat. Complut. 26(1), 147-181 (2013)

21. Erhardt, A.: Calderón-Zygmund theory for parabolic obstacle problems with nonstandard growth. Adv. Nonlinear Anal. 3(1), 15-44 (2014)

22. Erhardt, A.H.: Existence and gradient estimates in parabolic obstacle problems with nonstandard growth, Ph.D. thesis, Friedrich-Alexander University Erlangen-Nuremberg, (2013)

23. Erhardt, A.H.: Existence of solutions to parabolic problems with nonstandard growth and irregular obstacles. Adv. Differ. Equ. 21(5-6), 463-504 (2016)

24. Erhardt, A.H.: Higher integrability for solutions to parabolic problems with irregular obstacles and nonstandard growth. J. Math. Anal. Appl. 435(2), 1772-1803 (2016)

25. Erhardt, A.H.: Compact embedding for $p(x, t)$-Sobolev spaces and existence theory to parabolic equations with $p(x, t)$-growth. Rev. Mat. Complut. 30(1), 35-61 (2017)

26. Erhardt, A.H.: The stability of parabolic problems with nonstandard $p(x, t)$-growth. Mathematics 5(4), 14 (2017)

27. Feireisl, E.: Mathematical analysis of fluids in motion: from well-posedness to model reduction. Rev. Mat. Complut. 26(2), 299-340 (2013) 
28. Franzina, G., Lindqvist, P.: An eigenvalue problem with variable exponents. Nonlinear Anal. 85, 1-16 (2013)

29. Gao, W., Guo, B.: Existence and localization of weak solutions of nonlinear parabolic equations with variable exponent of nonlinearity. Ann. Mat. Pura Appl. (4) 191(3), 551-562 (2012)

30. Gerstenmayer, A., Jüngel, A.: Analysis of a degenerate parabolic cross-diffusion system for ion transport. J. Math. Anal. Appl. 461(1), 523-543 (2018)

31. Giaquinta, M., Modica, G.: Partial regularity of minimizers of quasiconvex integrals. Ann. Inst. H. Poincaré Anal. Non Linéaire 3(3), 185-208 (1986)

32. Guo, B., Gao, W.: Study of weak solutions for parabolic equations with nonstandard growth conditions. J. Math. Anal. Appl. 374(2), 374-384 (2011)

33. Hamburger, C.: Regularity of differential forms minimizing degenerate elliptic functionals. J. Reine Angew. Math. 431, 7-64 (1992)

34. Harjulehto, P., Hästö, P., Latvala, V., Toivanen, O.: Critical variable exponent functionals in image restoration. Appl. Math. Lett. 26(1), 56-60 (2013)

35. Hsieh, C.-Y.: Global existence of solutions for the Poisson-Nernst-Planck system with steric effects. Nonlinear Anal. Real World Appl. 50, 34-54 (2019)

36. Jüngel, A., Zamponi, N.: Qualitative behavior of solutions to cross-diffusion systems from population dynamics. J. Math. Anal. Appl. 440(2), 794-809 (2016)

37. Lair, A.V., Oxley, M.E.: A necessary and sufficient condition for global existence for a degenerate parabolic boundary value problem. J. Math. Anal. Appl. 221(1), 338-348 (1998)

38. Li, Y.: Global boundedness of weak solution in an attraction-repulsion chemotaxis system with $p$ -Laplacian diffusion. Nonlinear Anal. Real World Appl. 51, 102933, 18 (2020)

39. Liu, B., Dong, M.: A nonlinear diffusion problem with convection and anisotropic nonstandard growth conditions. Nonlinear Anal. Real World Appl. 48, 383-409 (2019)

40. Liu, Y., Zhang, Z., Zhu, L.: Global existence and blowup for a quasilinear parabolic equations with nonlinear gradient absorption. Adv. Differ. Equ. 24(3-4), 229-256 (2019)

41. Nirenberg, L.: On elliptic partial differential equations. Ann. Scuola Norm. Sup. Pisa Cl. Sci. (3) 13, 115-162 (1959)

42. Ok, J.: Harnack inequality for a class of functionals with non-standard growth via De Giorgi's method. Adv. Nonlinear Anal. 7(2), 167-182 (2018)

43. Pan, N., Zhang, B., Cao, J.: Weak solutions for parabolic equations with $p(x)$-growth. Electron. J. Differ. Equ. 209, 15 (2016)

44. Prasath, V.B.S., Vorotnikov, D.: On time adaptive critical variable exponent vectorial diffusion flows and their applications in image processing I: analysis. Nonlinear Anal. 168, 176-197 (2018)

45. Roubíček, T.: Nonlinear Partial Differential Equations with Applications, 2nd edn. International Series of Numerical Mathematics, vol. 153. Birkhäuser, Basel (2013)

46. Ružička, M.: Modeling, mathematical and numerical analysis of electrorheological fluids. Appl. Math. 49(6), 565-609 (2004)

47. Shangerganesh, L., Gurusamy, A., Balachandran, K.: Weak solutions for nonlinear parabolic equations with variable exponents. Commun. Math. 25(1), 55-70 (2017)

48. Singer, T., Vestberg, M.: Local boundedness of weak solutions to the diffusive wave approximation of the shallow water equations. J. Differ. Equ. 266(6), 3014-3033 (2019)

49. Winkert, P., Zacher, R.: Global a priori bounds for weak solutions to quasilinear parabolic equations with nonstandard growth. Nonlinear Anal. 145, 1-23 (2016)

50. Winther, R.: Error estimates for a Galerkin approximation of a parabolic control problem. Ann. Mat. Pura Appl. (4) 117, 173-206 (1978)

Publisher's Note Springer Nature remains neutral with regard to jurisdictional claims in published maps and institutional affiliations. 